Article

\title{
The Relationships of Habitat Conditions, Height Level, and Geographical Position with Fruit and Seed Traits in Populations of Invasive Vine Echinocystis lobata (Cucurbitaceae) in Central and Eastern Europe
}

\author{
Kinga Kostrakiewicz-Gierałt 1,*DD, Artur Pliszko ${ }^{2}$, Beata Barabasz-Krasny ${ }^{3}$, Anna Bomanowska ${ }^{4}$, \\ Zygmunt Dajdok ${ }^{5}{ }^{(D}$, Zigmantas Gudžinskas ${ }^{6}{ }^{(D}$, Marek Kucharczyk ${ }^{7}$, tukasz Maćkowiak ${ }^{8}$, Jakub Majk ${ }^{5}$ (D), \\ Katarzyna Możdżeń ${ }^{9} \mathbb{D}$, Monika Podgórska ${ }^{10}$, Mindaugas Rasimavičius ${ }^{11}\left(\mathbb{D}\right.$, Agnieszka Rewicz ${ }^{12}$ (D), \\ Ewa Szczęśniak ${ }^{5}$, Tomasz Wójcik ${ }^{13}$ (i) and Alina Stachurska-Swakoń ${ }^{14}$ (D)
}

check for updates

Citation: Kostrakiewicz-Gierałt, K.; Pliszko, A.; Barabasz-Krasny, B.; Bomanowska, A.; Dajdok, Z.; Gudžinskas, Z.; Kucharczyk, M.; Maćkowiak, Ł.; Majk, J.; Możdżeń, K.; et al. The Relationships of Habitat Conditions, Height Level, and Geographical Position with Fruit and Seed Traits in Populations of Invasive Vine Echinocystis lobata (Cucurbitaceae) in Central and Eastern Europe. Forests 2022, 13, 256. https://doi.org/10.3390/f13020256

Academic Editor: Luís González

Received: 14 October 2021

Accepted: 4 February 2022

Published: 6 February 2022

Publisher's Note: MDPI stays neutral with regard to jurisdictional claims in published maps and institutional affiliations.

Copyright: (C) 2022 by the authors. Licensee MDPI, Basel, Switzerland. This article is an open access article distributed under the terms and conditions of the Creative Commons Attribution (CC BY) license (https:// creativecommons.org/licenses/by/ $4.0 /)$.
1 Department of Tourism Geography and Ecology, Institute of Tourism, Faculty of Tourism and Recreation, University of Physical Education in Cracow, 31-571 Kraków, Poland

2 Department of Taxonomy, Phytogeography and Palaeobotany, Institute of Botany, Faculty of Biology, Jagiellonian University, Gronostajowa 3, 30-387 Kraków, Poland; artur.pliszko@uj.edu.pl

3 Department of Botany, Institute of Biology, Faculty of Exact and Natural Sciences, Pedagogical University of Cracow, Podchorążych 2, 30-084 Kraków, Poland; beata.barabasz-krasny@up.krakow.pl

4 Department of Geobotany and Plant Ecology, Faculty of Biology and Environmental Protection, University of Lodz, Banacha 12/16, 90-237 Łódź, Poland; anna.bomanowska@biol.uni.lodz.pl

5 Department of Botany, Institute of Environmental Biology, University of Wrocław, Kanonia 6/8, 50-328 Wrocław, Poland; zygmunt.dajdok@uwr.edu.pl (Z.D.); jakub.majk@gmail.com (J.M.); ewa.szczesniak@uwr.edu.pl (E.S.)

6 Nature Research Centre, Laboratory of Flora and Geobotany, Institute of Botany, Žaliuju Ežerų 49, 12000 Vilnius, Lithuania; zigmantas.gudzinskas@gamtc.lt

7 Department of Zoology and Nature Conservation, Institute of Biological Sciences, Maria Curie-Skłodowska University, 20-033 Lublin, Poland; marek.kucharczyk@mail.umcs.pl

8 Kowalewo 6, 64-061 Kamieniec, Poland; lukaszmackowiak123@gmail.com

9 Department of Plant Physiology, Institute of Biology, Faculty of Exact and Natural Sciences, Pedagogical University of Cracow, Podchorążych 2, 30-084 Kraków, Poland; katarzyna.mozdzen@up.krakow.pl

10 Department of Environmental Biology, Institute of Biology, Faculty of Exact and Natural Sciences, Jan Kochanowski University, Uniwersytecka 7, 25-406 Kielce, Poland; monika.podgorska@ujk.edu.pl

11 Life Science Centre, Institute of Biosciences, Vilnius University, Saulètekio Av. 7, LT-10257 Vilnius, Lithuania; mindaugas.rasimavicius@gf.vu.lt

12 Department of Biogeography, Paleoecology and Nature Conservation, Faculty of Biology and Environmental Protection, University of Lodz, Banacha 1/3, 90-237 Łódź, Poland; agnieszka.rewicz@biol.uni.lodz.pl

13 Department of Nature Conservation and Landscape Ecology, Institute of Agricultural Sciences, Land Management and Environmental Protection, University of Rzeszów, Zelwerowicza 4, 35-601 Rzeszów, Poland; twojcik@ur.edu.pl

14 Department of Plant Ecology, Institute of Botany, Jagiellonian University, Gronostajowa 3 , 30-387 Kraków, Poland; alina.stachurska-swakon@uj.edu.pl

* Correspondence: kinga.kostrakiewicz@awf.krakow.pl

Abstract: In climbing plants, fruits can be formed at different heights, depending on the height of external support. However, the effect of height on fruit and seed traits in invasive vines of Cucurbitaceae has not been intensively studied so far. In Europe, Echinocystis lobata, a North American member of Cucurbitaceae, is considered one of the most abundant invasive alien plants spreading in natural riparian forests, thickets, and tall herbs, whereas it is a rare species in urban woodlands. In this study, we tested the variability of selected fruit and seed traits of E. lobata in connection with habitat origin (natural, semi-natural, and anthropogenic), habitat type (using the EUNIS habitat classification), height (the distance between the fruit and the ground), and geographical position. The study was conducted in 2018 in 65 sites located in Latvia, Lithuania, Poland, and Slovakia. In each study site, a random sample of 10 fresh mature fruits of $E$. lobata was collected from subsequent $0.50 \mathrm{~m}$ intervals of height. The length, the width, and the weight of the fresh fruits, as well as 
the total number of seeds and number of undeveloped seeds per fruit, were examined. Moreover, the well-developed seeds from selected study sites were weighed. The statistical analysis showed the significant effect of habitat origin, habitat type, and height on most of the studied fruit and seed traits. The largest range of height (from 0.00 to $4.00 \mathrm{~m}$ ) was observed in two types of habitats (F-heathland, scrub, and tundra; and G-woodland, forest, and other wooded land). The total number of seeds per fruit was positively correlated with the length, width, and weight of the fresh fruits. The fruits were a little heavier and bigger in natural habitats located in the northeast of the study area. The distribution of fruits at different heights may contribute to better dispersal of seeds by animals and wind, and may also better protect the seeds from being eaten by granivorous animals. The defence against seed-eating animals is expected to be stronger in semi-natural habitats, as well as on banks and shores of inland surface waters, where the seed production is the highest. The influence of seed traits on seed germination and seedling survival in various habitats and their importance in the invasiveness of E. lobata require further study.

Keywords: climbing plants; fruit and seed size; invasive alien species; morphological variability

\section{Introduction}

Climbing plants use external support (usually neighbouring plants) to grow vertically for better light acquisition [1]. They occur mostly in forests from the tropics to the boreal zones in both the northern and southern hemispheres; however, their greatest diversity is found in the tropical zone [2,3]. Climbing plants have been recognized in many plant families, especially in Apocynaceae, Bignoniaceae, Celastraceae, Convolvulaceae, Cucurbitaceae, Dioscoreaceae, Fabaceae, Menispermaceae, Rubiaceae, and Vitaceae [2-4]. They have evolved diverse strategies allowing them to attach to the external support by forming structures such as twining stems and branches, tendrils (modified leaves, leaflets, stipules, inflorescences, branches, or stems), hooks, thorns, and adventitious roots [3-5]. Based on their life forms, climbing plants can be divided into woody lianas, herbaceous or subwoody vines, woody hemiepiphytes, and herbaceous epiphytes and hemiepiphytes [2]. In comparison with woody lianas, the distribution of herbaceous climbers is not affected by disturbance, but is dependent on the availability of external support. Herbaceous climbers grow in places with low light availability, but plenty of external support, and require magnesium for the formation of flexible stems [6]. Unlike trees, climbing plants invest little in thickening their stems and branches, using a large proportion of their resources to produce additional leaves as well as for reproduction [3]. In tropical forests, many climbing plants form deep root systems and may avoid competing with trees by avoiding drought stress through tapping deeper stores of water [3]. Some experiments have shown that climbing plants generally colonise nutrient-rich patches of soil much more quickly and with much less investment in root biomass than trees [3]. Consequently, climbing capacity is considered an important factor facilitating the invasion success of alien plants in forest communities [7-9]. There are some interesting similarities and differences between native and non-native climbing plants in patterns of distribution and seed dispersal. In Michigan, USA, for example, both native and non-native climbing species show a pattern of decreasing species richness with increasing latitude. However, native climbing species are $63 \%$ abiotically and $37 \%$ biotically dispersed, while non-native species are $62 \%$ abiotic and $38 \%$ biotic. Moreover, biotically dispersed species are more broadly distributed among Michigan counties than abiotic species [10].

Vines of Cucurbitaceae are commonly cultivated as ornamental, edible, and medicinal plants [11,12]. Unfortunately, many of them easily escape from their cultivation sites and become established and invasive in new areas $[13,14]$. Successful invasion of alien vines of Cucurbitaceae can be explained by their fast growth and various methods of dispersal. For example, S. angulatus has become an invasive weed in some European and Asian countries because of its seed dispersal with sowing material (harvest equipment for maize 
and soybean). Moreover, fruits of $S$. angulatus can be easily dispersed by flowing waters as well as by animals (small mammals and birds) and humans (attachment to clothes, agricultural practices, and transport) [15-17]. Coccinia grandis (L.) Voigt., by contrast, has become a noxious weed in Hawaii owing to its seed dispersal by birds and mammals $[18,19]$. Moreover, C. grandis can propagate vegetatively by fragmentation of tuberous rootstock and aerial shoots. According to Chun [20], fragments of C. grandis shoots grow roots readily at the nodes when they touch the soil. Another example, Thladiantha dubia Bunge, has become a dangerous and hard-to-eradicate weed in the Republic of Bashkortostan in Russia owing to its intensive vegetative propagation by fragmentation of tubers during mechanical soil treatment [21].

Biological invasions are one of the most serious threats to biodiversity and cultural heritage in the world [22,23]. Studies on the impact of habitat conditions on the production and dispersal of seeds in invasive alien plants allow a better understanding of their geographical distribution [24] and adaptability to new habitats [9], as well as enable the identification of habitats particularly sensitive to invasion [25]. Plant height, seed weight, and seed morphology are some of the most important traits influencing abiotic seed dispersal [26]. In climbing plants, the fruits can be formed at different heights, depending on the height of external support. This ability has a great influence on the efficiency of seed dispersal [27]. However, the effect of height on fruit and seed traits in invasive vines of Cucurbitaceae has not been intensively studied so far. In this study, we focused on Echinocystis lobata (Michx.) Torr. \& A. Gray, one of the most rapidly spreading invasive vines of Cucurbitaceae in Central and Eastern Europe in recent years [28-32]. We aimed to investigate (i) the effect of habitat origin (natural, semi-natural, or anthropogenic) on fruit size and number of seeds, (ii) the effect of habitat type (using the EUNIS classification [33]) and height level on fruit size and number of seeds, (iii) the relationship between fruit size and number of seeds, (iv) the effect of height level on the fresh and dry mass of seeds, (v) the relationship between fruit size and geographic position, and (vi) the relationship between seed size and geographic position.

\section{Materials and Methods}

\subsection{Study Species}

Echinocystis lobata, the wild cucumber, an annual, monoecious vine of the Cucurbitaceae, is native to temperate North America [34,35]. It occurs east of the Rocky Mountains, and mostly north of the Ohio River in the United States and in southern Canada, growing in riparian forests and thickets, river and ditch banks, waste places, and fencerows [36,37]. It was introduced into Europe at the end of the 19th century and the beginning of the 20th century as an ornamental and medicinal plant [28,29]. According to Bagiand Böszörményi [38], it was also introduced into Europe accidentally with cotton shipments. During the 20th century, E. lobata escaped from gardens, becoming a naturalized species in anthropogenic, semi-natural, and natural habitats. Nowadays, it is considered invasive in many European countries, especially in Central and Eastern Europe [28-31,39]. Populations of invasive E. lobata have been recorded in anthropogenic habitats (e.g., abandoned allotments, roadsides, railway embankments, waste dumps, and ditches), as well as in natural habitats (e.g., riparian forests, thickets, swamps, and riverbanks) [28-30,40-44].

E. lobata is a fast-growing and light-demanding vine adapted to moist and nutrient-rich habitats [35]. As an invasive species, it negatively affects the diversity, density, and morphological traits of native plants by strong competition for light, especially in the communities of Convolvuletalia sepium R.Tx., Alnenion glutinosae-incanae Oberd., and Salicion albae R.Tx. $[9,45,46]$. It climbs over the vegetation using long tendrils ( $20 \mathrm{~cm}$ or more), which are its modified lateral branches [47]. Sometimes willows, poplars, and marsh elms are almost completely covered by plexuses of climbing shoots of E. lobata [40]. The plant is $5 \mathrm{~m}$ or more long (up to $12 \mathrm{~m}$ ), forming protandrous, unisexual flowers arranged in a panicle inflorescence. Each inflorescence is composed of numerous male flowers (20-100) and one to four female flowers located at the base of the inflorescence [29,35-37]. In Central 
Europe, the flowering of E. lobata usually occurs from July to September and the flowers are pollinated by insects, but are also self-fertile [29]. The seeds are mature and shed 4-6 weeks after flowering when the fruit (pepo) dries out and the pericarp at the distal end splits and peels back to reveal the open mouths of two locules [35]. Selected morphological features of E. lobata are presented in Figure 1.
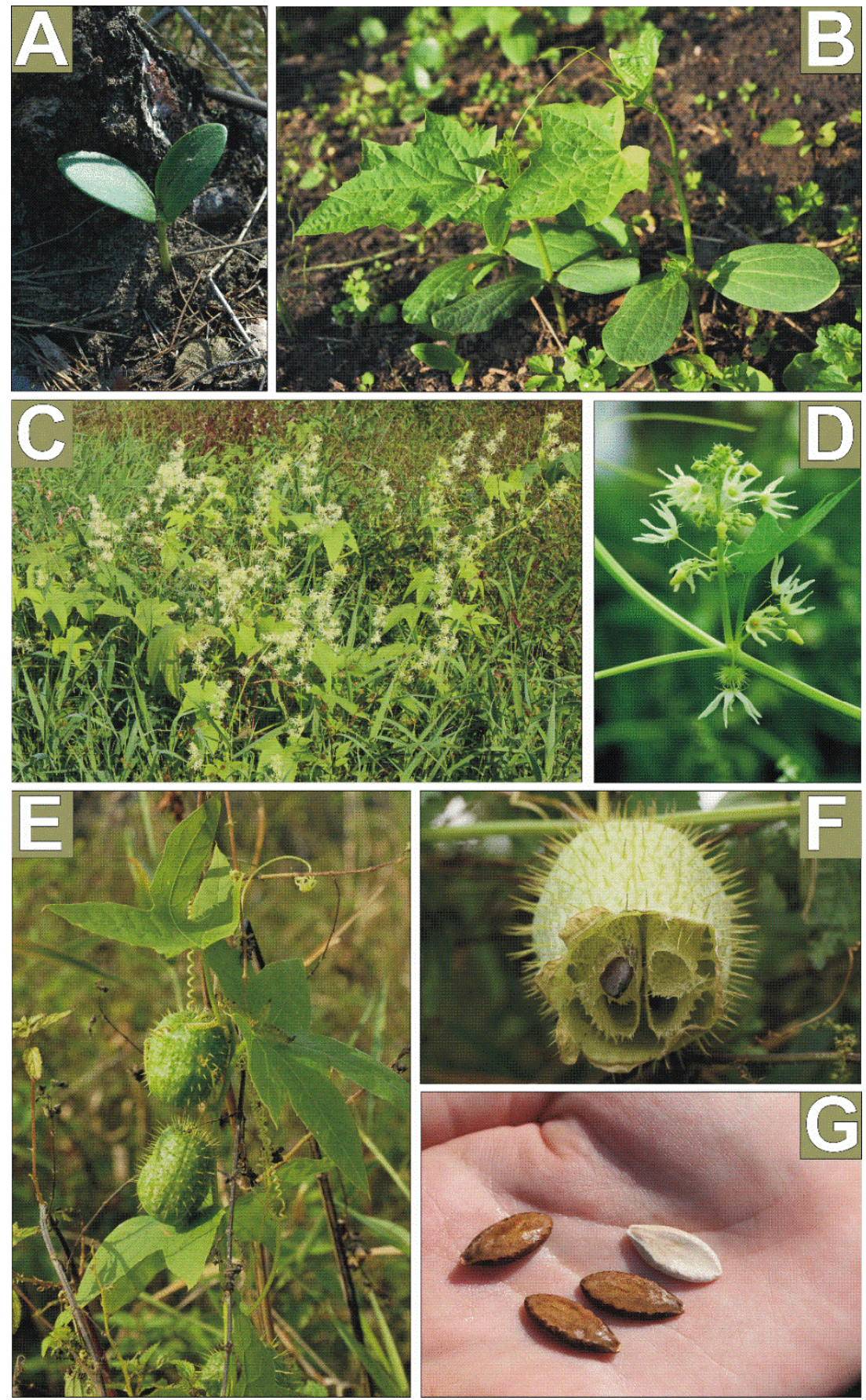

Figure 1. Morphological characteristics of Echinocystis lobata: (A)—seedling, (B)—young specimens with first leaves and tendrils, (C) - mature individual in a typical waterside habitat, (D) - fragment of the shoot with flowers, (E) — fruits (pepos), (F)—pen fruit at the stage of seed release, (G) — seeds (photographed by Z. Dajdok). 


\subsection{Study Area}

The investigations were conducted at 65 study sites located in Latvia, Lithuania, Poland, and Slovakia (Figure 2). The altitude of the localities ranged from $13 \mathrm{~m}$ a.s.l. to $304 \mathrm{~m}$ a.s.l. Based on the EUNIS habitat classification [33], the study sites represented the following habitat types: inland surface waters (code C); mires, bogs, and fens (D); grasslands and lands dominated by forbs, mosses, or lichens (E); heathland, scrub, and tundra (F); woodland, forest, and other wooded lands $(\mathrm{G})$; inland unvegetated or sparsely vegetated habitats $(\mathrm{H})$; regularly or recently cultivated agricultural, horticultural, and domestic habitats (I); constructed, industrial, and other artificial habitats (J); and habitat complexes $(X)$. The study sites included 25 habitat types; the greatest number of study sites represented riparian and gallery woodland (G1.1) and almond willow-osier scrub (F9.121), while the lowest number comprised domestic gardens of villages and urban peripheries (X25). The study sites included natural (43), semi-natural (3), and anthropogenic habitats (19) as three groups of habitat origin. In each study site, plant cover, light availability, and soil $\mathrm{pH}$ were estimated. The percentage of plant cover (with an interval of $5 \%$ ) was visually estimated in plots of $25 \mathrm{~m}^{2}$ considering three vegetation layers (A-tree layer, Bshrub layer, $\mathrm{C}$-herb layer). Considerable values for plant cover in the tree, shrub, and herb layers were recorded in most forest habitats such as riparian and gallery woodlands. In contrast, a low plant cover percentage solely in the herbaceous vegetation layer was recorded in many anthropogenic habitats. The light availability was estimated using a five-grade scale: $1-$ full shade (0-20\% of light availability), 2 -substantial shade (21-40\% of light availability), 3-moderate shade (41-60\% of light availability), 4-low shade (61-80\% of light availability), 5-full sun (81-100\% of light availability). The scale was created by calculating the mean plant coverage in the A, B, and C layers, and then subtracting the obtained value from $100 \%$ insolation. Most of the study sites were represented by low shaded and moderately shaded conditions. A fully shaded condition was not observed. The soil $\mathrm{pH}$ was measured using electronic devices and ranged from 5.0 to 8.0. Most of the study sites showed the soil $\mathrm{pH}$ from 6.1 to 7.0. The detailed characteristics of the study sites are presented in Table A1.

\subsection{Fruit and Seed Sampling}

The investigations were carried out in September and October 2018. In each study site, a random sample of 10 mature fruits was collected from the subsequent height ranges: $0.00 \mathrm{~m}-0.50 \mathrm{~m}, 0.51 \mathrm{~m}-1.00 \mathrm{~m}, 1.01 \mathrm{~m}-1.50 \mathrm{~m}$, and so on. Next, the length and the width of the fruits (excluding spinules) were measured using an electronic caliper with an accuracy of $0.1 \mathrm{~cm}$. Simultaneously, the fresh fruits were weighed using an electronic scale with an accuracy of $0.1 \mathrm{~g}$. Then, the total number of seeds in each fruit was counted. Moreover, the number of undeveloped (empty, deformed) seeds was noted. Simultaneously, properly developed seeds from the chosen study sites were weighed separately using an electronic scale with an accuracy of $0.01 \mathrm{~g}$. The seeds were weighed per fruit and the mean mass of one seed was calculated. Altogether, 491 seeds were weighed. The seeds were kept in paper trays, allowing open-air drying. Subsequently, 199 portions containing 10 dry seeds collected at a certain location and at a defined height level were weighed on an electronic weighing scale with an accuracy of $0.001 \mathrm{~g}$. After laboratory analyses, the portions of seeds were stored separately in labelled paper bags. 


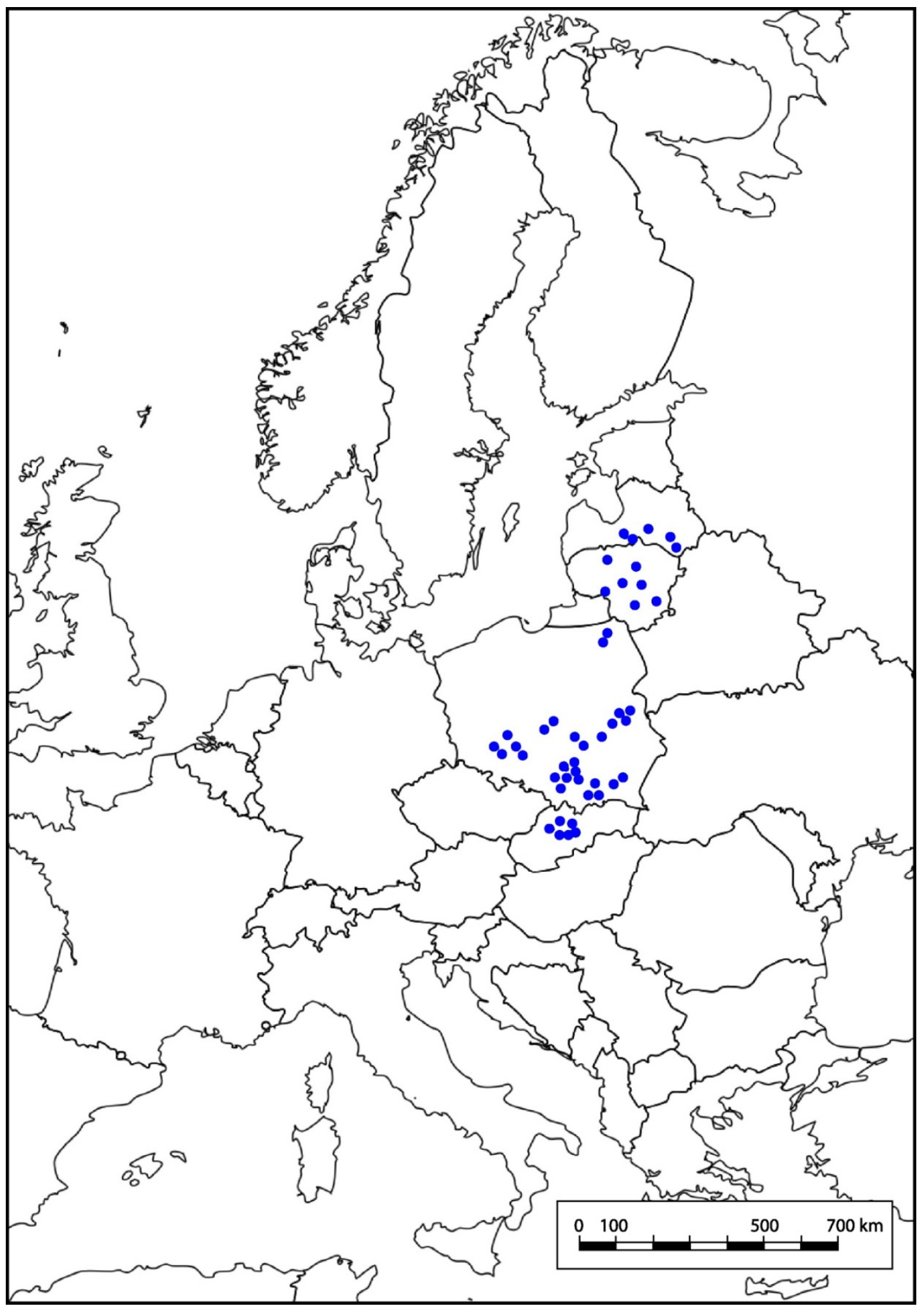

Figure 2. Distribution of sampled populations of Echinocystis lobata.

\subsection{Statistical Analyses}

The arithmetic mean (x) and standard deviation (SD) of length, width, and fresh weight of fruits, as well as the total number of seeds per fruit and number of undeveloped seeds in habitats with different origin and type as well as at diverse height intervals, were calculated. One way ANOVA followed by a post-hoc Tukey test was applied to test the 
statistical significance of differences in (i) the length, (ii) the width, and (iii) the fresh weight of fruits, as well as (iv) the total number of seeds per fruit and (v) the number of undeveloped seeds per fruit, considering (i) habitats with different origin, (ii) different types of habitats, and (iii) different height intervals (levels). Then, the two-way ANOVA was used to test the statistical significance of differences in the abovementioned traits of fruits, sampled at various height levels in different habitat types. Before using ANOVA, the normal distribution of untransformed data was checked by the Kolmogorov-Smirnov test, while the homogeneity of variance was tested using the Levene test at a significance level of $p<0.05$. The correlation between (i) the length, (ii) the weight, and (iii) the fresh weight of fruits and the total number of seeds, as well as the number of undeveloped seeds, was tested using the Pearson coefficient at a level of $p<0.05$. The impact of height level on the weight of fresh seeds was tested by applying one-way ANOVA (the data meet assumptions of the normal distribution and variance homogeneity), whereas the impact of height level on the weight of dry seeds was tested using the Kruskal-Wallis H test because of the small number of records in some groups. The variability in fruit and seed size with the geographic position was tested using the F-test or Kruskal-Wallis test when the normality assumption was not met. In the case of fresh fruits, the weight, length, and width were tested with geographical locations. These parameters were tested separately for different height levels and origin of habitat. Similarly, the seed size (in the meaning of biomass) was tested. The CCA (Canonical Correspondence Analysis) was used to find the influence of environmental variables on fruit size variability. The significance of geographical position, light intensity, soil $\mathrm{pH}$, habitat type, and habitat origin was also tested. The mean of fruit parameters for all height levels per locality was taken. MVSP 3.22 software [48] was used for this purpose. For other tests, STATISTICA 13.0 software was involved [49].

\section{Results}

\subsection{The Effect of Habitat Origin on Fruit and Seed Traits}

The length and width of the fruits achieved the greatest values in natural habitats, while the fresh weight of fruits was much greater in natural and semi-natural habitats than in anthropogenic ones (Table 1). The total number of seeds per fruit was the greatest in semi-natural habitats and the lowest in anthropogenic habitats, whereas the number of undeveloped seeds was similar in all groups of habitats (Table 1).

Table 1. The fruit and seed traits (mean $\pm \mathrm{SD}$ ) of Echinocystis lobata in anthropogenic (A), seminatural $(\mathrm{SN})$, and natural habitats $(\mathrm{N})$.

\begin{tabular}{|c|c|c|c|c|c|c|}
\hline $\begin{array}{c}\text { Habitat } \\
\text { Origin }\end{array}$ & $\begin{array}{c}\text { Number of } \\
\text { Records }\end{array}$ & $\begin{array}{l}\text { Fruit Length } \\
\qquad(\mathrm{cm})\end{array}$ & $\begin{array}{l}\text { Fruit Width } \\
\quad(\mathrm{cm})\end{array}$ & $\begin{array}{l}\text { Fruit Fresh } \\
\text { Weight (g) }\end{array}$ & $\begin{array}{l}\text { Total Number of } \\
\text { Seeds per Fruit }\end{array}$ & $\begin{array}{l}\text { Number of Undeveloped } \\
\text { Seeds per Fruit }\end{array}$ \\
\hline A & 566 & $\begin{array}{c}4.17 \\
( \pm 0.59)^{\mathrm{a}}\end{array}$ & $\begin{array}{c}3.18 \\
( \pm 0.41)^{a}\end{array}$ & $\begin{array}{c}12.95 \\
( \pm 4.92)^{\mathrm{a}}\end{array}$ & $\begin{array}{c}3.86 \\
( \pm 1.04)^{\mathrm{a}}\end{array}$ & $\begin{array}{c}0.56 \\
( \pm 1.00)^{\mathrm{a}}\end{array}$ \\
\hline $\mathrm{N}$ & 1758 & $\begin{aligned} & 4.70 \\
&( \pm 0.63)^{b}\end{aligned}$ & $\begin{aligned} & 3.43 \\
&( \pm 0.84)^{b}\end{aligned}$ & $\begin{aligned} & 15.92 \\
&( \pm 4.79)^{b}\end{aligned}$ & $\begin{aligned} & 4.04 \\
&( \pm 0.89)^{b}\end{aligned}$ & $\begin{array}{c}0.55 \\
( \pm 1.16)^{\mathrm{a}}\end{array}$ \\
\hline $\mathrm{SN}$ & 103 & $\begin{array}{c}4.45 \\
( \pm 0.82)^{\mathrm{c}}\end{array}$ & $\begin{array}{c}3.08 \\
( \pm 0.46)^{a}\end{array}$ & $\begin{array}{c}14.17 \\
( \pm 6.19) \mathrm{b}\end{array}$ & $\begin{array}{c}4.33 \\
( \pm 0.99)^{\mathrm{c}}\end{array}$ & $\begin{array}{c}0.45 \\
( \pm 1.02)^{\mathrm{a}}\end{array}$ \\
\hline \multirow{2}{*}{\multicolumn{2}{|c|}{$\begin{array}{l}\text { F value } \\
(d f=2) \\
p \text { value }\end{array}$}} & 151.85 & 30.91 & 80.79 & 14.59 & 0.38 \\
\hline & & $p<0.001$ & $p<0.001$ & $p<0.001$ & $p<0.001$ & $p=0.67$ \\
\hline
\end{tabular}

The diverse letters in superscripts mean the statistical differences between anthropogenic, semi-natural, and natural habitats.

\subsection{The Effect of Habitat Type on Fruit and Seed Traits}

The effect of the habitat type was significant in the case of the length of fruits $(\mathrm{F}=24.45$, $\mathrm{df}=8, p<0.001)$. The greatest mean value of the fruit length $(5.16 \mathrm{~cm})$ was noted in mires, bogs, and fens (habitat group D), while the lowest one $(4.02 \mathrm{~cm}$ ) was found in regularly or recently cultivated agricultural, horticultural, and domestic habitats (I) (Table 2). 
The statistical analysis showed that the width of fresh fruits reaching 3.15-3.97 cm did not vary remarkably $(\mathrm{F}=0.35, \mathrm{df}=8, p=0.49)$. Moreover, the weight of fresh fruits measuring $12.05-21.61 \mathrm{~g}$ did not differ between habitats $(\mathrm{F}=0.02, \mathrm{df}=8, p=0.83)$. The total number of seeds varied significantly between habitats $(\mathrm{F}=22.65, \mathrm{df}=8, p<0.001)$, ranging from 3.43 in habitat complexes $(\mathrm{X})$ to 4.50 in inland surface water habitats $(\mathrm{C})$. Similarly, the number of undeveloped seeds differed significantly $(\mathrm{F}=10.12, \mathrm{df}=8, p<0.001)$, ranging from 0.18 in habitat complexes $(\mathrm{X})$ to 0.70 in woodland, forest, and other wooded lands $(\mathrm{G})$ (Table 2).

Table 2. The fruit and seed traits (mean $\pm \mathrm{SD}$ ) of Echinocystis lobata in inland surface waters (C); mires, bogs, and fens (D); grasslands and lands dominated by forbs, mosses, or lichens (E); heathland, scrub, and tundra $(\mathrm{F})$; woodland, forest, and other wooded land $(\mathrm{G})$; inland unvegetated or sparsely vegetated habitats $(\mathrm{H})$; regularly or recently cultivated agricultural, horticultural, and domestic habitats(I); constructed, industrial, and other artificial habitats (J); habitat complexes (X).

\begin{tabular}{|c|c|c|c|c|c|c|}
\hline $\begin{array}{l}\text { Habitat } \\
\text { Type }\end{array}$ & $\begin{array}{c}\text { Number of } \\
\text { Records }\end{array}$ & $\begin{array}{l}\text { Fruit Length } \\
\quad(\mathrm{cm})\end{array}$ & $\begin{array}{l}\text { Fruit Width } \\
\qquad(\mathrm{cm})\end{array}$ & $\begin{array}{l}\text { Fruit Fresh } \\
\text { Weight (g) }\end{array}$ & $\begin{array}{l}\text { Total Number of } \\
\text { Seeds per Fruit }\end{array}$ & $\begin{array}{l}\text { Number of Undeveloped } \\
\text { Seeds per Fruit }\end{array}$ \\
\hline C & 100 & $\begin{array}{c}4.51 \\
( \pm 0.94)\end{array}$ & $\begin{array}{c}3.24 \\
( \pm 0.57)\end{array}$ & $\begin{array}{c}15.77 \\
( \pm 6.01)\end{array}$ & $\begin{array}{c}4.50 \\
( \pm 0.85)\end{array}$ & $\begin{array}{c}0.64 \\
( \pm 1.15)\end{array}$ \\
\hline D & 30 & $\begin{array}{c}5.16 \\
( \pm 0.28) \\
\end{array}$ & $\begin{array}{c}3.97 \\
( \pm 0.28)\end{array}$ & $\begin{array}{c}21.69 \\
( \pm 4.47)\end{array}$ & $\begin{array}{c}4.33 \\
( \pm 0.54)\end{array}$ & $\begin{array}{c}0.36 \\
( \pm 0.76)\end{array}$ \\
\hline E & 430 & $\begin{array}{c}4.42 \\
( \pm 0.70)\end{array}$ & $\begin{array}{c}3.33 \\
( \pm 0.40)\end{array}$ & $\begin{array}{c}14.48 \\
( \pm 5.28)\end{array}$ & $\begin{array}{c}4.19 \\
( \pm 0.87)\end{array}$ & $\begin{array}{c}0.48 \\
( \pm 0.98)\end{array}$ \\
\hline $\mathrm{F}$ & 474 & $\begin{array}{c}4.92 \\
( \pm 0.53)\end{array}$ & $\begin{array}{c}3.42 \\
( \pm 0.33)\end{array}$ & $\begin{array}{c}16.28 \\
( \pm 4.49)\end{array}$ & $\begin{array}{c}4.17 \\
( \pm 0.54)\end{array}$ & $\begin{array}{c}0.35 \\
( \pm 0.71)\end{array}$ \\
\hline $\mathrm{G}$ & 919 & $\begin{array}{c}4.67 \\
( \pm 0.60)\end{array}$ & $\begin{array}{c}3.45 \\
( \pm 1.11)\end{array}$ & $\begin{array}{c}15.96 \\
( \pm 4.64)\end{array}$ & $\begin{array}{c}3.88 \\
( \pm 1.03)\end{array}$ & $\begin{array}{c}0.70 \\
( \pm 1.38)\end{array}$ \\
\hline $\mathrm{H}$ & 29 & $\begin{array}{c}4.72 \\
( \pm 0.35)\end{array}$ & $\begin{array}{c}3.15 \\
( \pm 0.23)\end{array}$ & $\begin{array}{c}16.56 \\
( \pm 3.55)\end{array}$ & $\begin{array}{c}4.38 \\
( \pm 0.37)\end{array}$ & $\begin{array}{c}0.65 \\
( \pm 0.67)\end{array}$ \\
\hline I & 96 & $\begin{array}{c}4.02 \\
( \pm 0.55)\end{array}$ & $\begin{array}{c}3.15 \\
( \pm 0.44)\end{array}$ & $\begin{array}{c}12.59 \\
( \pm 4.27)\end{array}$ & $\begin{array}{c}3.91 \\
( \pm 1.06)\end{array}$ & $\begin{array}{c}0.30 \\
( \pm 0.63)\end{array}$ \\
\hline $\mathrm{J}$ & 289 & $\begin{array}{c}4.09 \\
( \pm 0.53)\end{array}$ & $\begin{array}{c}3.15 \\
( \pm 0.41)\end{array}$ & $\begin{array}{c}12.05 \\
( \pm 4.42)\end{array}$ & $\begin{array}{c}3.83 \\
( \pm 1.07)\end{array}$ & $\begin{array}{c}0.62 \\
( \pm 1.24)\end{array}$ \\
\hline$x$ & 60 & $\begin{array}{c}4.09 \\
( \pm 0.61)\end{array}$ & $\begin{array}{c}3.26 \\
( \pm 0.47)\end{array}$ & $\begin{array}{c}12.77 \\
( \pm 5.99)\end{array}$ & $\begin{array}{c}3.43 \\
( \pm 0.92)\end{array}$ & $\begin{array}{c}0.18 \\
( \pm 0.53)\end{array}$ \\
\hline
\end{tabular}

\subsection{The Effect of Height Level on Fruit and Seed Traits}

The statistical analysis showed the effect of height level on most of the fruit traits. The statistically significant differences $(\mathrm{F}=2.47 \mathrm{df}=7, p<0.01)$ presented the length of fruits achieving the greatest values at a height of 3.01-3.50 m and 3.51-4.00 m. Similarly, the weight of fruits varied remarkably $(\mathrm{F}=2.94, \mathrm{df}=7, p<0.01)$ and reached the greatest values at a height of $2.51-3.00 \mathrm{~m}$. The greatest numbers of total seeds per fruit $(\mathrm{F}=10.25$, $\mathrm{df}=7, p<0.001)$ and undeveloped seeds per fruit $(\mathrm{F}=10.90, \mathrm{df}=7, p<0.001)$ were found at the lowest height intervals. The width of fruits did not differ $(F=0.36, d f=7$, $p=0.92$ ) (Table 3). The one-way ANOVA test showed that the fresh weight of seeds varied significantly between height levels $(\mathrm{F}=2.48, \mathrm{df}=7, p<0.01)$. Remarkable differences were noticed between fresh seeds collected at a height of 2.01-2.50 m, achieving the greatest weight, and those collected at a height of 3.51-4.00 m, achieving the lowest weight (Table 4). Furthermore, the Kruskal-Wallis H test showed no differences in the mass of dry seeds collected at different height levels $(\mathrm{H}=11.80, p=0.1)$ (Table 5). 
Table 3. The fruit and seed traits (mean $\pm \mathrm{SD}$ ) of Echinocystis lobata at different height levels.

\begin{tabular}{|c|c|c|c|c|c|c|}
\hline $\begin{array}{l}\text { Height } \\
\text { Level (m) }\end{array}$ & $\begin{array}{c}\text { Number of } \\
\text { Records }\end{array}$ & $\begin{array}{l}\text { Fruit Length } \\
(\mathrm{cm})\end{array}$ & $\begin{array}{l}\text { Fruit Width } \\
\quad(\mathrm{cm})\end{array}$ & $\begin{array}{l}\text { Fruit Fresh } \\
\text { Weight (g) }\end{array}$ & $\begin{array}{l}\text { Total Number of } \\
\text { Seeds per Fruit }\end{array}$ & $\begin{array}{c}\text { Number of Undeveloped } \\
\text { Seeds per Fruit }\end{array}$ \\
\hline $0.00-0.50$ & 469 & $\begin{array}{c}4.52 \\
( \pm 0.69)\end{array}$ & $\begin{array}{c}3.32 \\
( \pm 0.45)\end{array}$ & $\begin{array}{l}15.49 \\
( \pm 5.33)\end{array}$ & $\begin{array}{c}4.08 \\
( \pm 0.84)\end{array}$ & $\begin{array}{c}0.89 \\
( \pm 1.42)\end{array}$ \\
\hline $0.51-1.00$ & 387 & $\begin{array}{c}4.61 \\
( \pm 0.73)\end{array}$ & $\begin{array}{c}3.35 \\
( \pm 0.41)\end{array}$ & $\begin{array}{c}15.65 \\
( \pm 5.62)\end{array}$ & $\begin{array}{c}4.12 \\
( \pm 0.97)\end{array}$ & $\begin{array}{c}0.43 \\
( \pm 1.00)\end{array}$ \\
\hline $1.01-1.50$ & 517 & $\begin{array}{c}4.53 \\
( \pm 0.64) \\
\end{array}$ & $\begin{array}{c}3.38 \\
( \pm 1.44) \\
\end{array}$ & $\begin{array}{c}14.40 \\
( \pm 5.15) \\
\end{array}$ & $\begin{array}{c}3.88 \\
( \pm 1.10) \\
\end{array}$ & $\begin{array}{c}0.60 \\
( \pm 1.21) \\
\end{array}$ \\
\hline $1.51-2.00$ & 397 & $\begin{array}{c}4.53 \\
( \pm 0.64)\end{array}$ & $\begin{array}{c}3.40 \\
( \pm 0.45)\end{array}$ & $\begin{array}{c}14.94 \\
( \pm 4.86)\end{array}$ & $\begin{array}{c}4.03 \\
( \pm 0.80)\end{array}$ & $\begin{array}{c}0.42 \\
( \pm 1.02)\end{array}$ \\
\hline $2.01-2.50$ & 296 & $\begin{array}{c}4.55 \\
( \pm 0.66)\end{array}$ & $\begin{array}{c}3.36 \\
( \pm 0.36)\end{array}$ & $\begin{array}{c}15.13 \\
( \pm 4.04)\end{array}$ & $\begin{array}{c}4.11 \\
( \pm 0.79)\end{array}$ & $\begin{array}{c}0.45 \\
( \pm 1.02)\end{array}$ \\
\hline $2.51-3.00$ & 216 & $\begin{array}{c}4.60 \\
( \pm 0.66) \\
\end{array}$ & $\begin{array}{c}3.36 \\
( \pm 0.34) \\
\end{array}$ & $\begin{array}{c}15.69 \\
( \pm 4.02) \\
\end{array}$ & $\begin{array}{c}4.05 \\
( \pm 0.75) \\
\end{array}$ & $\begin{array}{c}0.29 \\
( \pm 0.59) \\
\end{array}$ \\
\hline $3.01-3.50$ & 82 & $\begin{array}{c}4.74 \\
( \pm 0.67) \\
\end{array}$ & $\begin{array}{c}3.33 \\
( \pm 0.39) \\
\end{array}$ & $\begin{array}{c}15.23 \\
( \pm 4.72) \\
\end{array}$ & $\begin{array}{c}3.97 \\
( \pm 0.81) \\
\end{array}$ & $\begin{array}{c}0.21 \\
( \pm 0.56)\end{array}$ \\
\hline $3.51-4.00$ & 63 & $\begin{array}{c}4.77 \\
( \pm 0.55)\end{array}$ & $\begin{array}{c}3.35 \\
( \pm 0.33)\end{array}$ & $\begin{array}{c}15.25 \\
( \pm 3.55)\end{array}$ & $\begin{array}{c}3.19 \\
( \pm 1.51)\end{array}$ & $\begin{array}{c}0.73 \\
( \pm 1.43)\end{array}$ \\
\hline
\end{tabular}

Table 4. The fresh weight of the seeds (mean \pm SD) of Echinocystis lobata sampled at different height levels in selected study sites.

\begin{tabular}{ccc}
\hline Height Level $(\mathbf{m})$ & Number of Seeds & Fresh Mass of Seeds (mg) \\
\hline $0.00-0.50$ & 30 & $1.53( \pm 0.42)$ \\
$0.51-1.00$ & 100 & $1.55( \pm 0.45)$ \\
$1.01-1.50$ & 117 & $1.59( \pm 0.57)$ \\
$1.51-2.00$ & 77 & $1.65( \pm 0.44)$ \\
$2.01-2.50$ & 60 & $1.82( \pm 0.39)$ \\
$2.51-3.00$ & 47 & $1.61( \pm 0.42)$ \\
$3.01-3.50$ & 30 & $1.55( \pm 0.53)$ \\
$3.51-4.00$ & 30 & $1.50( \pm 0.42)$ \\
\hline
\end{tabular}

Table 5. The dry weight of 10 seeds (mean \pm SD) of Echinocystis lobata (one record) sampled at different height levels in selected study sites.

\begin{tabular}{ccc}
\hline Height Level $(\mathbf{m})$ & Number of Records & Dry Mass of Seeds $(\mathbf{g})$ \\
\hline $0.00-0.50$ & 42 & $0.27( \pm 0.03)$ \\
$0.51-1.00$ & 37 & $0.27( \pm 0.03)$ \\
$1.01-1.51$ & 38 & $0.29( \pm 0.04)$ \\
$1.51-2.00$ & 29 & $0.28( \pm 0.04)$ \\
$2.01-2.50$ & 23 & $0.28( \pm 0.04)$ \\
$2.51-3.00$ & 20 & $0.29( \pm 0.04)$ \\
$3.01-3.50$ & 6 & $0.30( \pm 0.03)$ \\
$3.51-4.00$ & 4 & $0.31( \pm 0.03)$ \\
\hline
\end{tabular}

3.4. The Relationships of Habitat Origin, Habitat Type, and Height Level with Fruit and Seed Traits

A remarkable interaction between habitat type and height level on fruit length $(\mathrm{F}=2.61$, $\mathrm{df}=42, p<0.001$ ) was evidenced (Table 6). The greatest length of fruit was observed in mires, bogs, and fens (D) at a height of $0.00-0.50 \mathrm{~m}$ and in forest habitats (G) at a height of 3.01-3.50 m, while the lowest fruit length was noted in habitat complexes (X) at a height of 3.01-3.50 m. The Tukey test showed that samples from the forest $(\mathrm{G})$ and shrubland habitats (F) collected at the heights of 1.51-2.00 m, 2.01-2.50 m, and 2.51-3.00 m differed significantly 
from samples collected in agricultural, horticultural, and domestic habitats (I); constructed, industrial, and other artificial habitats $(\mathrm{J})$; and habitat complexes $(\mathrm{X})$, irrespective of the height level (Table 6).

Table 6. The length $(\mathrm{cm})$ of the fruits(mean $\pm \mathrm{SD})$ of Echinocystis lobata at different height levels in inland surface waters (C); mires, bogs, and fens (D); grasslands and lands dominated by forbs, mosses, or lichens (E); heathland, scrub, and tundra (F); woodland, forest, and other wooded land (G); inland unvegetated or sparsely vegetated habitats $(\mathrm{H})$; regularly or recently cultivated agricultural, horticultural, and domestic habitats (I); constructed, industrial, and other artificial habitats (J); habitat complexes $(\mathrm{X})$.

\begin{tabular}{|c|c|c|c|c|c|c|c|c|c|}
\hline \multirow{2}{*}{$\begin{array}{c}\text { Height } \\
\text { Level (m) }\end{array}$} & \multicolumn{9}{|c|}{ Habitat Type } \\
\hline & $C$ & D & E & $\mathbf{F}$ & G & $\mathbf{H}$ & I & $\mathbf{J}$ & $x$ \\
\hline $0.00-0.50$ & $\begin{array}{c}4.38 \\
( \pm 0.79)\end{array}$ & $\begin{array}{c}5.22 \\
( \pm 0.28)\end{array}$ & $\begin{array}{c}4.53 \\
( \pm 0.74)\end{array}$ & $\begin{array}{c}4.75 \\
( \pm 0.52)\end{array}$ & $\begin{array}{c}4.70 \\
( \pm 0.73)\end{array}$ & $\begin{array}{c}4.56 \\
( \pm 0.38)\end{array}$ & $\begin{array}{c}3.96 \\
( \pm 0.67)\end{array}$ & $\begin{array}{c}4.21 \\
( \pm 0.40)\end{array}$ & \\
\hline $0.51-1.00$ & $\begin{array}{c}4.79 \\
( \pm 1.34)\end{array}$ & & $\begin{array}{c}4.58 \\
( \pm 0.58)\end{array}$ & $\begin{array}{c}5.05 \\
( \pm 0.52)\end{array}$ & $\begin{array}{c}4.68 \\
( \pm 0.66)\end{array}$ & $\begin{array}{c}4.91 \\
( \pm 0.33)\end{array}$ & $\begin{array}{c}4.03 \\
( \pm 0.53)\end{array}$ & $\begin{array}{c}4.01 \\
( \pm 0.62)\end{array}$ & $\begin{array}{c}3.76 \\
( \pm 0.46)\end{array}$ \\
\hline $1.01-1.51$ & $\begin{array}{c}4.56 \\
( \pm 0.25) \\
\end{array}$ & $\begin{array}{c}5.20 \\
( \pm 0.25) \\
\end{array}$ & $\begin{array}{c}4.52 \\
( \pm 0.61) \\
\end{array}$ & $\begin{array}{c}4.92 \\
( \pm 0.55) \\
\end{array}$ & $\begin{array}{c}4.57 \\
( \pm 0.61) \\
\end{array}$ & $\begin{array}{c}4.68 \\
( \pm 0.26) \\
\end{array}$ & $\begin{array}{c}4.04 \\
( \pm 0.43) \\
\end{array}$ & $\begin{array}{c}4.08 \\
( \pm 0.50) \\
\end{array}$ & $\begin{array}{c}4.08 \\
( \pm 0.32) \\
\end{array}$ \\
\hline $1.51-2.00$ & $\begin{array}{c}4.50 \\
( \pm 0.29)\end{array}$ & $\begin{array}{c}5.07 \\
( \pm 0.30)\end{array}$ & $\begin{array}{c}4.19 \\
( \pm 0.56)\end{array}$ & $\begin{array}{c}4.95 \\
( \pm 0.51)\end{array}$ & $\begin{array}{c}4.64 \\
( \pm 0.57)\end{array}$ & & $\begin{array}{c}3.95 \\
( \pm 0.68)\end{array}$ & $\begin{array}{c}4.10 \\
( \pm 0.64)\end{array}$ & $\begin{array}{c}4.51 \\
( \pm 0.59)\end{array}$ \\
\hline $2.01-2.50$ & & & $\begin{array}{c}4.20 \\
( \pm 0.80)\end{array}$ & $\begin{array}{c}4.84 \\
( \pm 0.54)\end{array}$ & $\begin{array}{c}4.70 \\
( \pm 0.49)\end{array}$ & & $\begin{array}{c}4.13 \\
( \pm 0.48)\end{array}$ & $\begin{array}{c}3.63 \\
( \pm 0.52)\end{array}$ & $\begin{array}{c}4.54 \\
( \pm 0.78)\end{array}$ \\
\hline $2.51-3.00$ & & & $\begin{array}{c}4.25 \\
( \pm 0.86) \\
\end{array}$ & $\begin{array}{c}4.93 \\
( \pm 0.51) \\
\end{array}$ & $\begin{array}{c}4.74 \\
( \pm 0.50) \\
\end{array}$ & & $\begin{array}{c}4.05 \\
( \pm 0.37) \\
\end{array}$ & $\begin{array}{c}4.17 \\
( \pm 0.37) \\
\end{array}$ & $\begin{array}{c}4.09 \\
( \pm 0.33) \\
\end{array}$ \\
\hline $3.01-3.50$ & & & & $\begin{array}{c}5.22 \\
( \pm 0.61)\end{array}$ & $\begin{array}{c}4.77 \\
( \pm 0.52)\end{array}$ & & & & $\begin{array}{c}3.60 \\
( \pm 0.44)\end{array}$ \\
\hline $3.51-4.00$ & & & & $\begin{array}{c}5.17 \\
( \pm 0.22)\end{array}$ & $\begin{array}{c}4.68 \\
( \pm 0.49)\end{array}$ & & & & \\
\hline
\end{tabular}

The statistical analysis showed no influence of the interaction of habitat type and height level on fruit width $(\mathrm{F}=1.11, \mathrm{df}=42, p=0.28)$ (Table 7). The influence of the interaction of habitat type and height level on fruit weight was significant $(\mathrm{F}=2.61, \mathrm{df}=42$, $p<0.001)$. The lowest fresh weight of fruit $(8.41 \mathrm{~g})$ was noticed in habitat complexes $(\mathrm{X})$ at a height of 3.01-3.50 m, while the greatest one $(23.60 \mathrm{~g})$ was recorded in mires, bogs, and fens (D) at a height of 0.00-0.50 $\mathrm{m}$ (Table 8). The Tukey test confirmed significant differences among samples derived from mires, bogs, and fens growing at a height of $0.00-0.51 \mathrm{~m}$ and samples taken from other habitats regardless of height level. Moreover, samples derived from woodlands $(\mathrm{G})$ and heathland, scrub, and tundra (F) differed remarkably from cultivated agricultural, horticultural, and domestic habitats (I), and habitat complexes (X), irrespective of the height level (Table 8). The influence of the interaction of habitat type and height level on the total number of seeds per fruit was also significant $(\mathrm{F}=2.43, \mathrm{df}=42$, $p<0.001)$. The lowest numbers of seeds per fruit amounted to 2.70 and 2.92, noticed in habitat complexes $(X)$ at a height of 3.01-3.50 m and wooded habitats $(G)$ at a height of 3.51-4.00 m, respectively (Table 9). The Tukey test confirmed significant differences among the abovementioned samples, as well as among samples taken from other habitats, irrespective of height level (Table 9). Moreover, the statistical analysis showed the significant influence of the interaction of habitat type and height level on the number of undeveloped seeds per fruit $(\mathrm{F}=2.43, \mathrm{df}=42, p<0.001)$. The greatest number of undeveloped seeds (1.58) was noticed in constructed, industrial, and other artificial habitats (J) at a height of $0.00-0.50 \mathrm{~m}$, and it was significantly greater than in other sites (Table 10). The seed size did not differ between natural and anthropogenic habitats measured at different height levels; however, a trend with smaller seeds in anthropogenic habitats was noticed (Figure 3). 
Table 7. The width $(\mathrm{cm})$ of the fruits (mean $\pm \mathrm{SD}$ ) of Echinocystis lobata at different height levels in inland surface waters (C); mires, bogs, and fens (D); grasslands and lands dominated by forbs, mosses, or lichens (E); heathland, scrub, and tundra (F); woodland, forest, and other wooded land (G); inland unvegetated or sparsely vegetated habitats $(\mathrm{H})$; regularly or recently cultivated agricultural, horticultural and domestic habitats (I); constructed, industrial, and other artificial habitats (J); habitat complexes (X).

\begin{tabular}{|c|c|c|c|c|c|c|c|c|c|}
\hline \multirow{2}{*}{$\begin{array}{c}\text { Height } \\
\text { Level (m) }\end{array}$} & \multicolumn{9}{|c|}{ Habitat Type } \\
\hline & $\mathrm{C}$ & D & E & F & $G$ & $\mathbf{H}$ & I & $\mathbf{J}$ & $x$ \\
\hline $0.00-0.50$ & $\begin{array}{c}3.03 \\
( \pm 0.63)\end{array}$ & $\begin{array}{c}3.92 \\
( \pm 0.26)\end{array}$ & $\begin{array}{c}3.43 \\
( \pm 0.42)\end{array}$ & $\begin{array}{c}3.40 \\
( \pm 0.32)\end{array}$ & $\begin{array}{c}3.40 \\
( \pm 0.42)\end{array}$ & $\begin{array}{c}3.15 \\
( \pm 0.27)\end{array}$ & $\begin{array}{c}2.91 \\
( \pm 0.49)\end{array}$ & $\begin{array}{c}3.28 \\
( \pm 0.37)\end{array}$ & \\
\hline $0.51-1.00$ & $\begin{array}{c}3.57 \\
( \pm 0.53) \\
\end{array}$ & & $\begin{array}{c}3.35 \\
( \pm 0.37) \\
\end{array}$ & $\begin{array}{c}3.49 \\
( \pm 0.34) \\
\end{array}$ & $\begin{array}{c}3.41 \\
( \pm 0.39) \\
\end{array}$ & $\begin{array}{c}3.27 \\
( \pm 0.20) \\
\end{array}$ & $\begin{array}{c}3.45 \\
( \pm 0.52) \\
\end{array}$ & $\begin{array}{c}3.04 \\
( \pm 0.40) \\
\end{array}$ & $\begin{array}{c}2.93 \\
( \pm 0.34)\end{array}$ \\
\hline $1.01-1.51$ & $\begin{array}{c}3.28 \\
( \pm 0.35)\end{array}$ & $\begin{array}{c}3.92 \\
( \pm 0.36)\end{array}$ & $\begin{array}{c}3.33 \\
( \pm 0.33)\end{array}$ & $\begin{array}{c}3.38 \\
( \pm 0.35)\end{array}$ & $\begin{array}{c}3.53 \\
( \pm 2.29)\end{array}$ & $\begin{array}{c}3.03 \\
( \pm 0.15)\end{array}$ & $\begin{array}{c}3.15 \\
( \pm 0.35)\end{array}$ & $\begin{array}{c}3.09 \\
( \pm 0.37)\end{array}$ & $\begin{array}{c}3.32 \\
( \pm 0.24)\end{array}$ \\
\hline $1.51-2.00$ & $\begin{array}{c}3.59 \\
( \pm 0.13)\end{array}$ & $\begin{array}{c}4.07 \\
( \pm 0.18)\end{array}$ & $\begin{array}{c}3.26 \\
( \pm 0.40)\end{array}$ & $\begin{array}{c}3.47 \\
( \pm 0.32)\end{array}$ & $\begin{array}{c}3.46 \\
( \pm 0.49)\end{array}$ & & $\begin{array}{c}3.11 \\
( \pm 0.48)\end{array}$ & $\begin{array}{c}3.20 \\
( \pm 0.41)\end{array}$ & $\begin{array}{c}3.65 \\
( \pm 0.50)\end{array}$ \\
\hline $2.01-2.50$ & & & $\begin{array}{c}3.34 \\
( \pm 0.42)\end{array}$ & $\begin{array}{c}3.38 \\
( \pm 0.36)\end{array}$ & $\begin{array}{c}3.41 \\
( \pm 0.25)\end{array}$ & & $\begin{array}{c}3.32 \\
( \pm 0.33)\end{array}$ & $\begin{array}{c}2.88 \\
( \pm 0.59)\end{array}$ & $\begin{array}{c}3.58 \\
( \pm 0.32)\end{array}$ \\
\hline $2.51-3.00$ & & & $\begin{array}{c}3.26 \\
( \pm 0.46)\end{array}$ & $\begin{array}{c}3.43 \\
( \pm 0.29)\end{array}$ & $\begin{array}{c}3.39 \\
( \pm 0.29)\end{array}$ & & $\begin{array}{c}3.26 \\
( \pm 0.26)\end{array}$ & $\begin{array}{c}3.29 \\
( \pm 0.27)\end{array}$ & $\begin{array}{c}3.28 \\
( \pm 0.33) \\
\end{array}$ \\
\hline $3.01-3.50$ & & & & $\begin{array}{c}3.37 \\
( \pm 0.27)\end{array}$ & $\begin{array}{c}3.42 \\
( \pm 0.25)\end{array}$ & & & & $\begin{array}{c}2.79 \\
( \pm 0.35)\end{array}$ \\
\hline $3.51-4.00$ & & & & $\begin{array}{c}3.43 \\
( \pm 0.27)\end{array}$ & $\begin{array}{c}3.34 \\
( \pm 0.34)\end{array}$ & & & & \\
\hline
\end{tabular}

Table 8. The fresh weight (g) of the fruits (mean \pm SD) of Echinocystis lobata at different height levels in inland surface waters (C); mires, bogs, and fens (D); grasslands and lands dominated by forbs, mosses, or lichens $(\mathrm{E})$; heathland, scrub, and tundra $(\mathrm{F})$; woodland, forest, and other wooded land $(\mathrm{G})$; inland unvegetated or sparsely vegetated habitats $(\mathrm{H})$; regularly or recently cultivated agricultural, horticultural and domestic habitats (I); constructed, industrial, and other artificial habitats (J); habitat complexes (X).

\begin{tabular}{|c|c|c|c|c|c|c|c|c|c|}
\hline \multirow{2}{*}{$\begin{array}{c}\text { Height } \\
\text { Level (m) }\end{array}$} & \multicolumn{9}{|c|}{ Habitat Type } \\
\hline & $C$ & D & $E$ & F & G & $\mathrm{H}$ & I & $\mathbf{J}$ & $x$ \\
\hline $0.00-0.50$ & $\begin{array}{c}15.44 \\
( \pm 5.60) \\
\end{array}$ & $\begin{array}{c}23.60 \\
( \pm 6.25) \\
\end{array}$ & $\begin{array}{c}15.33 \\
( \pm 5.51) \\
\end{array}$ & $\begin{array}{c}15.98 \\
( \pm 4.63) \\
\end{array}$ & $\begin{array}{c}16.59 \\
( \pm 4.92) \\
\end{array}$ & $\begin{array}{c}15.43 \\
( \pm 3.05) \\
\end{array}$ & $\begin{array}{c}13.59 \\
( \pm 6.09) \\
\end{array}$ & $\begin{array}{c}13.20 \\
( \pm 4.72) \\
\end{array}$ & \\
\hline $0.51-1.00$ & $\begin{array}{c}18.17 \\
( \pm 7.71) \\
\end{array}$ & & $\begin{array}{c}16.54 \\
( \pm 5.99) \\
\end{array}$ & $\begin{array}{c}17.40 \\
( \pm 4.71) \\
\end{array}$ & $\begin{array}{c}15.67 \\
( \pm 5.32)\end{array}$ & $\begin{array}{c}18.77 \\
( \pm 3.85)\end{array}$ & $\begin{array}{c}13.03 \\
( \pm 3.02)\end{array}$ & $\begin{array}{c}11.37 \\
( \pm 3.85)\end{array}$ & $\begin{array}{c}10.88 \\
( \pm 3.27)\end{array}$ \\
\hline $1.01-1.51$ & $\begin{array}{c}15.12 \\
( \pm 6.07)\end{array}$ & $\begin{array}{c}21.94 \\
( \pm 3.07)\end{array}$ & $\begin{array}{c}13.62 \\
( \pm 5.01)\end{array}$ & $\begin{array}{c}16.22 \\
( \pm 5.09)\end{array}$ & $\begin{array}{c}14.97 \\
( \pm 4.95)\end{array}$ & $\begin{array}{c}15.36 \\
( \pm 2.78)\end{array}$ & $\begin{array}{c}12.30 \\
( \pm 3.48)\end{array}$ & $\begin{array}{c}11.30 \\
( \pm 4.53)\end{array}$ & $\begin{array}{c}12.06 \\
( \pm 2.24)\end{array}$ \\
\hline $1.51-2.00$ & $\begin{array}{c}13.93 \\
( \pm 2.41)\end{array}$ & $\begin{array}{c}19.53 \\
( \pm 2.60)\end{array}$ & $\begin{array}{c}13.22 \\
( \pm 4.31)\end{array}$ & $\begin{array}{c}15.63 \\
( \pm 3.44)\end{array}$ & $\begin{array}{c}16.29 \\
( \pm 5.01)\end{array}$ & & $\begin{array}{c}11.14 \\
( \pm 4.45)\end{array}$ & $\begin{array}{c}12.12 \\
( \pm 4.23)\end{array}$ & $\begin{array}{c}17.51 \\
( \pm 8.11)\end{array}$ \\
\hline $2.01-2.50$ & & & $\begin{array}{c}13.62 \\
( \pm 4.63)\end{array}$ & $\begin{array}{c}15.78 \\
( \pm 5.52)\end{array}$ & $\begin{array}{c}16.49 \\
( \pm 3.28)\end{array}$ & & $\begin{array}{c}12.95 \\
( \pm 2.87)\end{array}$ & $\begin{array}{c}8.85 \\
( \pm 2.82)\end{array}$ & $\begin{array}{c}14.91 \\
( \pm 7.98)\end{array}$ \\
\hline $2.51-3.00$ & & & $\begin{array}{c}13.54 \\
( \pm 4.60)\end{array}$ & $\begin{array}{c}17.36 \\
( \pm 3.10)\end{array}$ & $\begin{array}{c}16.51 \\
( \pm 3.54)\end{array}$ & & $\begin{array}{c}12.98 \\
( \pm 3.38)\end{array}$ & $\begin{array}{c}14.50 \\
( \pm 2.37)\end{array}$ & $\begin{array}{c}12.87 \\
( \pm 4.57)\end{array}$ \\
\hline $3.01-3.50$ & & & & $\begin{array}{c}15.00 \\
( \pm 3.56)\end{array}$ & $\begin{array}{c}16.63 \\
( \pm 4.13)\end{array}$ & & & & $\begin{array}{c}8.41 \\
( \pm 3.72)\end{array}$ \\
\hline $3.51-4.00$ & & & & $\begin{array}{c}16.59 \\
( \pm 1.73)\end{array}$ & $\begin{array}{c}14.96 \\
( \pm 3.78)\end{array}$ & & & & \\
\hline
\end{tabular}


Table 9. The number of seeds per fruit (mean \pm SD) of Echinocystis lobata at different height levels in inland surface waters (C); mires, bogs, and fens (D); grasslands and lands dominated by forbs, mosses, or lichens (E); heathland, scrub, and tundra (F); woodland, forest, and other wooded land (G); inland unvegetated or sparsely vegetated habitats $(\mathrm{H})$; regularly or recently cultivated agricultural, horticultural and domestic habitats (I); constructed, industrial, and other artificial habitats (J); habitat complexes (X).

\begin{tabular}{|c|c|c|c|c|c|c|c|c|c|}
\hline \multirow{2}{*}{$\begin{array}{c}\text { Height } \\
\text { Level (m) }\end{array}$} & \multicolumn{9}{|c|}{ Habitat Type } \\
\hline & $\mathrm{C}$ & D & E & F & $G$ & $\mathbf{H}$ & I & $\mathbf{J}$ & $x$ \\
\hline $0.00-0.50$ & $\begin{array}{c}4.42 \\
( \pm 0.99)\end{array}$ & $\begin{array}{c}4.60 \\
( \pm 0.51)\end{array}$ & $\begin{array}{c}4.00 \\
( \pm 0.71)\end{array}$ & $\begin{array}{c}4.30 \\
( \pm 0.59)\end{array}$ & $\begin{array}{c}4.00 \\
( \pm 0.77)\end{array}$ & $\begin{array}{c}4.22 \\
( \pm 0.46)\end{array}$ & $\begin{array}{c}3.91 \\
( \pm 1.26)\end{array}$ & $\begin{array}{c}3.92 \\
( \pm 0.99)\end{array}$ & \\
\hline $0.51-1.00$ & $\begin{array}{c}4.65 \\
( \pm 0.81) \\
\end{array}$ & & $\begin{array}{c}4.30 \\
( \pm 0.98) \\
\end{array}$ & $\begin{array}{c}4.28 \\
( \pm 0.68) \\
\end{array}$ & $\begin{array}{c}3.96 \\
( \pm 1.04) \\
\end{array}$ & $\begin{array}{c}4.70 \\
( \pm 0.44) \\
\end{array}$ & $\begin{array}{c}3.75 \\
( \pm 1.28) \\
\end{array}$ & $\begin{array}{c}3.96 \\
( \pm 0.87) \\
\end{array}$ & $\begin{array}{c}3.10 \\
( \pm 1.10)\end{array}$ \\
\hline $1.01-1.51$ & $\begin{array}{c}4.70 \\
( \pm 0.65)\end{array}$ & $\begin{array}{c}4.40 \\
( \pm 0.69)\end{array}$ & $\begin{array}{c}4.25 \\
( \pm 0.88)\end{array}$ & $\begin{array}{c}4.05 \\
( \pm 0.46)\end{array}$ & $\begin{array}{c}3.62 \\
( \pm 1.30)\end{array}$ & $\begin{array}{c}4.20 \\
( \pm 0.94)\end{array}$ & $\begin{array}{c}3.60 \\
( \pm 0.50)\end{array}$ & $\begin{array}{c}3.70 \\
( \pm 1.36)\end{array}$ & $\begin{array}{c}3.70 \\
( \pm 0.67)\end{array}$ \\
\hline $1.51-2.00$ & $\begin{array}{c}4.20 \\
( \pm 0.42)\end{array}$ & $\begin{array}{c}4.00 \\
( \pm 0.00)\end{array}$ & $\begin{array}{c}4.08 \\
( \pm 0.89)\end{array}$ & $\begin{array}{c}4.06 \\
( \pm 0.55)\end{array}$ & $\begin{array}{c}4.04 \\
( \pm 0.74)\end{array}$ & & $\begin{array}{c}4.20 \\
( \pm 1.47)\end{array}$ & $\begin{array}{c}3.86 \\
( \pm 0.95)\end{array}$ & $\begin{array}{c}4.00 \\
( \pm 0.81)\end{array}$ \\
\hline $2.01-2.50$ & & & $\begin{array}{c}4.16 \\
( \pm 0.97)\end{array}$ & $\begin{array}{c}4.26 \\
( \pm 0.57)\end{array}$ & $\begin{array}{c}4.12 \\
( \pm 0.84)\end{array}$ & & $\begin{array}{c}3.90 \\
( \pm 0.64)\end{array}$ & $\begin{array}{c}3.66 \\
( \pm 0.89)\end{array}$ & $\begin{array}{c}3.80 \\
( \pm 0.42)\end{array}$ \\
\hline $2.51-3.00$ & & & $\begin{array}{c}4.40 \\
( \pm 0.90)\end{array}$ & $\begin{array}{c}4.11 \\
( \pm 0.37)\end{array}$ & $\begin{array}{c}3.92 \\
( \pm 0.67) \\
\end{array}$ & & $\begin{array}{c}4.33 \\
( \pm 0.81)\end{array}$ & $\begin{array}{c}3.54 \\
( \pm 1.12)\end{array}$ & $\begin{array}{c}3.30 \\
( \pm 0.82) \\
\end{array}$ \\
\hline $3.01-3.50$ & & & & $\begin{array}{c}4.05 \\
( \pm 0.22)\end{array}$ & $\begin{array}{c}4.19 \\
( \pm 0.68)\end{array}$ & & & & $\begin{array}{c}2.70 \\
( \pm 1.05)\end{array}$ \\
\hline $3.51-4.00$ & & & & $\begin{array}{c}4.45 \\
( \pm 0.52)\end{array}$ & $\begin{array}{c}2.92 \\
( \pm 1.51)\end{array}$ & & & & \\
\hline
\end{tabular}

Table 10. The number of undeveloped seeds per fruit (mean \pm SD) of Echinocystis lobata at different height levels in inland surface waters (C); mires, bogs, and fens (D); grasslands and lands dominated by forbs, mosses, or lichens (E); heathland, scrub, and tundra $(\mathrm{F})$; woodland, forest, and other wooded land $(\mathrm{G})$; inland unvegetated or sparsely vegetated habitats $(\mathrm{H})$; regularly or recently cultivated agricultural, horticultural and domestic habitats (I); constructed, industrial, and other artificial habitats (J); habitat complexes (X).

\begin{tabular}{|c|c|c|c|c|c|c|c|c|c|}
\hline \multirow{2}{*}{$\begin{array}{c}\text { Height } \\
\text { Level (m) }\end{array}$} & \multicolumn{9}{|c|}{ Habitat Type } \\
\hline & $C$ & D & E & F & G & $\mathbf{H}$ & I & $\mathbf{J}$ & $X$ \\
\hline $0.00-0.50$ & $\begin{array}{c}1.04 \\
( \pm 1.42)\end{array}$ & $\begin{array}{c}0.40 \\
( \pm 0.96)\end{array}$ & $\begin{array}{c}0.50 \\
( \pm 1.04)\end{array}$ & $\begin{array}{c}0.33 \\
( \pm 0.63)\end{array}$ & $\begin{array}{c}1.04 \\
( \pm 1.61)\end{array}$ & $\begin{array}{c}0.55 \\
( \pm 0.72)\end{array}$ & $\begin{array}{c}0.41 \\
( \pm 0.59)\end{array}$ & $\begin{array}{c}1.58 \\
( \pm 1.73)\end{array}$ & \\
\hline $0.51-1.00$ & $\begin{array}{c}0.35 \\
( \pm 0.58)\end{array}$ & & $\begin{array}{c}0.47 \\
( \pm 0.95)\end{array}$ & $\begin{array}{c}0.28 \\
( \pm 0.56)\end{array}$ & $\begin{array}{c}0.63 \\
( \pm 1.37)\end{array}$ & $\begin{array}{c}0.80 \\
( \pm 0.63)\end{array}$ & $\begin{array}{c}0.25 \\
( \pm 0.46)\end{array}$ & $\begin{array}{c}0.19 \\
( \pm 0.56)\end{array}$ & $\begin{array}{c}0.20 \\
( \pm 0.63)\end{array}$ \\
\hline $1.01-1.51$ & $\begin{array}{c}0.25 \\
( \pm 0.71) \\
\end{array}$ & $\begin{array}{c}0.30 \\
( \pm 0.67) \\
\end{array}$ & $\begin{array}{c}0.58 \\
( \pm 1.11) \\
\end{array}$ & $\begin{array}{c}0.43 \\
( \pm 0.83) \\
\end{array}$ & $\begin{array}{c}0.99 \\
( \pm 1.38) \\
\end{array}$ & $\begin{array}{c}0.60 \\
( \pm 0.69)\end{array}$ & $\begin{array}{c}0.15 \\
( \pm 0.48) \\
\end{array}$ & $\begin{array}{c}0.10 \\
( \pm 0.30) \\
\end{array}$ & $\begin{array}{c}0.00 \\
( \pm 0.00)\end{array}$ \\
\hline $1.51-2.00$ & $\begin{array}{c}0.00 \\
( \pm 0.00)\end{array}$ & $\begin{array}{c}0.30 \\
( \pm 0.69)\end{array}$ & $\begin{array}{c}0.41 \\
( \pm 0.80)\end{array}$ & $\begin{array}{c}0.19 \\
( \pm 0.48)\end{array}$ & $\begin{array}{c}0.65 \\
( \pm 1.38)\end{array}$ & & $\begin{array}{c}0.40 \\
( \pm 0.94)\end{array}$ & $\begin{array}{c}0.29 \\
( \pm 0.75)\end{array}$ & $\begin{array}{c}0.00 \\
( \pm 0.00)\end{array}$ \\
\hline $2.01-2.50$ & & & $\begin{array}{c}0.52 \\
( \pm 1.05)\end{array}$ & $\begin{array}{c}0.54 \\
( \pm 0.99)\end{array}$ & $\begin{array}{c}0.48 \\
( \pm 1.16)\end{array}$ & & $\begin{array}{c}0.20 \\
( \pm 0.41)\end{array}$ & $\begin{array}{c}0.26 \\
( \pm 0.59)\end{array}$ & $\begin{array}{c}0.10 \\
( \pm 0.31)\end{array}$ \\
\hline $2.51-3.00$ & & & $\begin{array}{c}0.32 \\
( \pm 0.76)\end{array}$ & $\begin{array}{c}0.32 \\
( \pm 0.53)\end{array}$ & $\begin{array}{c}0.29 \\
( \pm 0.55)\end{array}$ & & $\begin{array}{c}0.50 \\
( \pm 0.83)\end{array}$ & $\begin{array}{c}0.09 \\
( \pm 0.30)\end{array}$ & $\begin{array}{c}0.10 \\
( \pm 0.31)\end{array}$ \\
\hline $3.01-3.50$ & & & & $\begin{array}{c}0.50 \\
( \pm 0.76)\end{array}$ & $\begin{array}{c}0.02 \\
( \pm 0.13)\end{array}$ & & & & $\begin{array}{c}0.70 \\
( \pm 0.94)\end{array}$ \\
\hline $3.51-4.00$ & & & & $\begin{array}{c}0.09 \\
( \pm 0.30)\end{array}$ & $\begin{array}{c}0.87 \\
( \pm 1.54)\end{array}$ & & & & \\
\hline
\end{tabular}




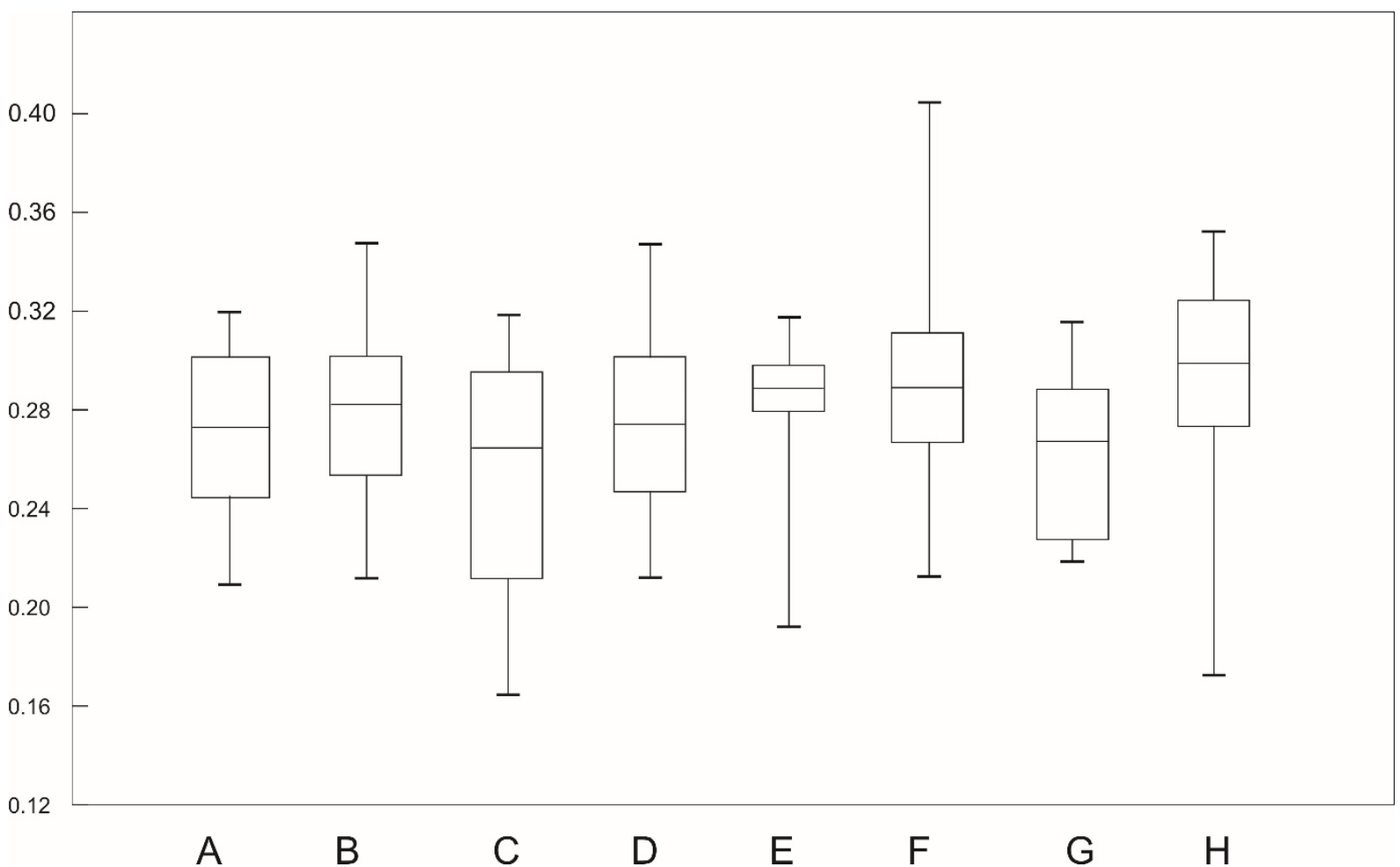

Figure 3. The mean of seed mass in pairs: anthropogenic $(A, C, E, G)$ and natural $(B, D, F, H)$ habitats at different height levels. A, B: 0.01-0.5 m, C, D: 0.51-1.0 m, E, F: $1.01-1.5 \mathrm{~m}, \mathrm{G}, \mathrm{H}: 1.51-2.0 \mathrm{~m}$. The differences are not significant (Kruskal-Wallis test, $p>0.05$ ).

\subsection{The Interdependence between the Traits of Fruits and Seeds}

The statistical analysis showed the occurrence of positive correlations among the length, width, and fresh weight of fruits. The total number of seeds was positively correlated with other fruit parameters, except for the number of undeveloped seeds. The number of undeveloped seeds was negatively correlated with the length and fresh weight of fruit, as well as with the total number of seeds. There was no correlation between the number of undeveloped seeds and the width of the fruit (Table 11).

Table 11. The Pearson coefficient $(p<0.05)$ between the length, the width, and the fresh weight of fruits, as well as the total number of seeds and number of undeveloped seeds in Echinocystis lobata. The statistically significant values are in bold.

\begin{tabular}{cccccc}
\hline & $\begin{array}{c}\text { Length of } \\
\text { Fruits }\end{array}$ & $\begin{array}{c}\text { Width of } \\
\text { Fruits }\end{array}$ & $\begin{array}{c}\text { Fresh Weight of } \\
\text { Fruits }\end{array}$ & $\begin{array}{c}\text { Total Number of } \\
\text { Seeds }\end{array}$ & $\begin{array}{c}\text { Number of } \\
\text { Undeveloped Seeds }\end{array}$ \\
\hline Length of fruits & 1.00 & $\mathbf{0 . 2 9}$ & $\mathbf{0 . 6 6}$ & $\mathbf{0 . 2 8}$ & $-\mathbf{0 . 0 7}$ \\
Width of fruits & & 1.00 & $\mathbf{0 . 3 5}$ & $\mathbf{0 . 1 0}$ & 0.01 \\
Fresh weight of fruits & & & 1.00 & $\mathbf{0 . 3 1}$ & $-\mathbf{0 . 1 0}$ \\
Total number of seeds & & & 1.00 & $-\mathbf{0 . 0 8}$ \\
$\quad$ Number of & & & & 1.00 \\
undeveloped seeds & & & & \\
\hline
\end{tabular}

\subsection{The Effect of Geographical Position on Fruit and Seed Traits}

The fruit size of $E$. lobata (in the meaning of fresh weight, length, and width) varied with the geographical position. The fruits were a little heavier and bigger in natural habitats located in the northeast of the study area (Figure 4). This correlation was statistically significant for all height levels; however, the biggest difference was for the level of 1.01-1.50 m. There was no such statistical correlation in anthropogenic habitats. Moreover, the correlation between the seed size and geographical position was statistically insignificant for both 
habitat origin and for height levels. The observed relation of geographical position and fruit size is the strongest one among the environmental conditions that were considered (Figure 5). However, the studied variables explained only $35 \%$ of the variability between the data.
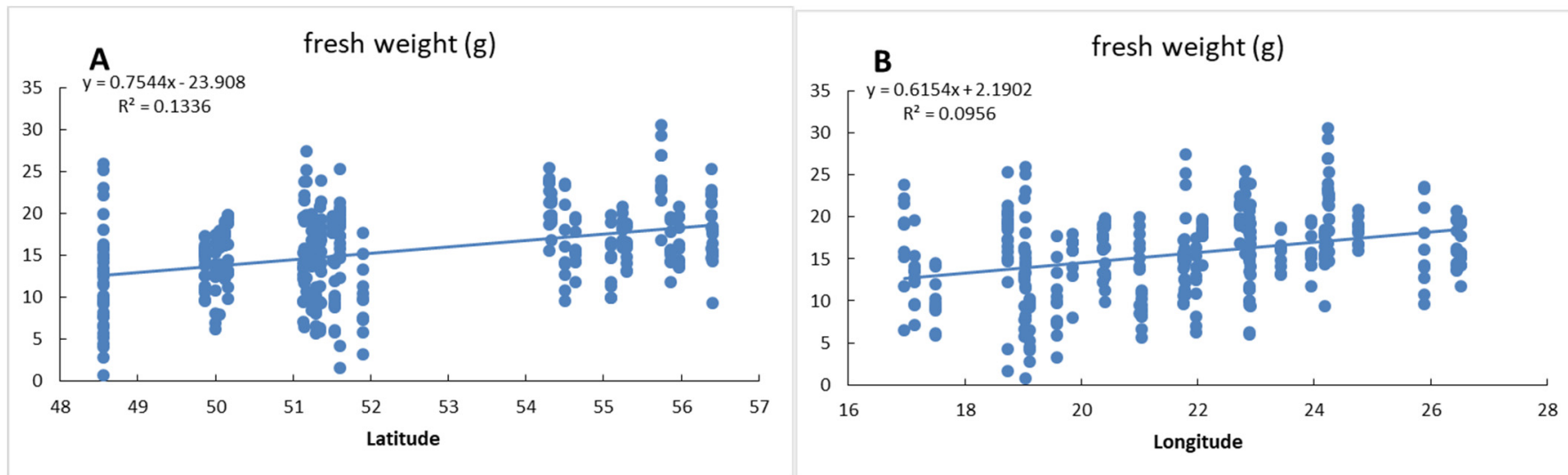

\section{C}

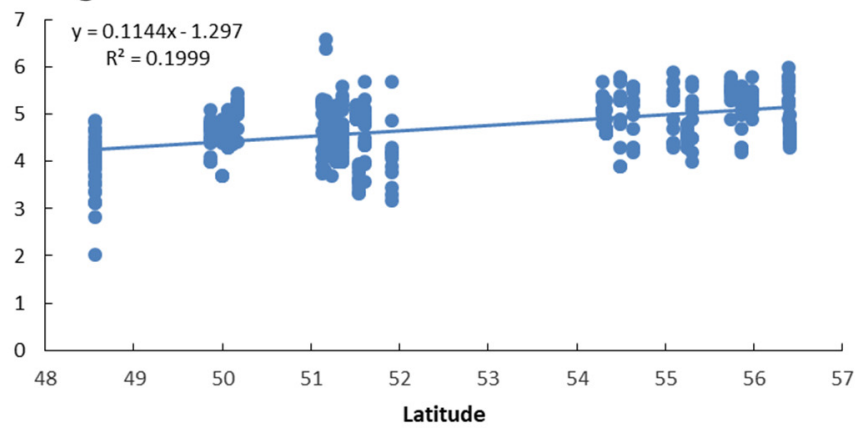

D

Length $(\mathrm{cm})$
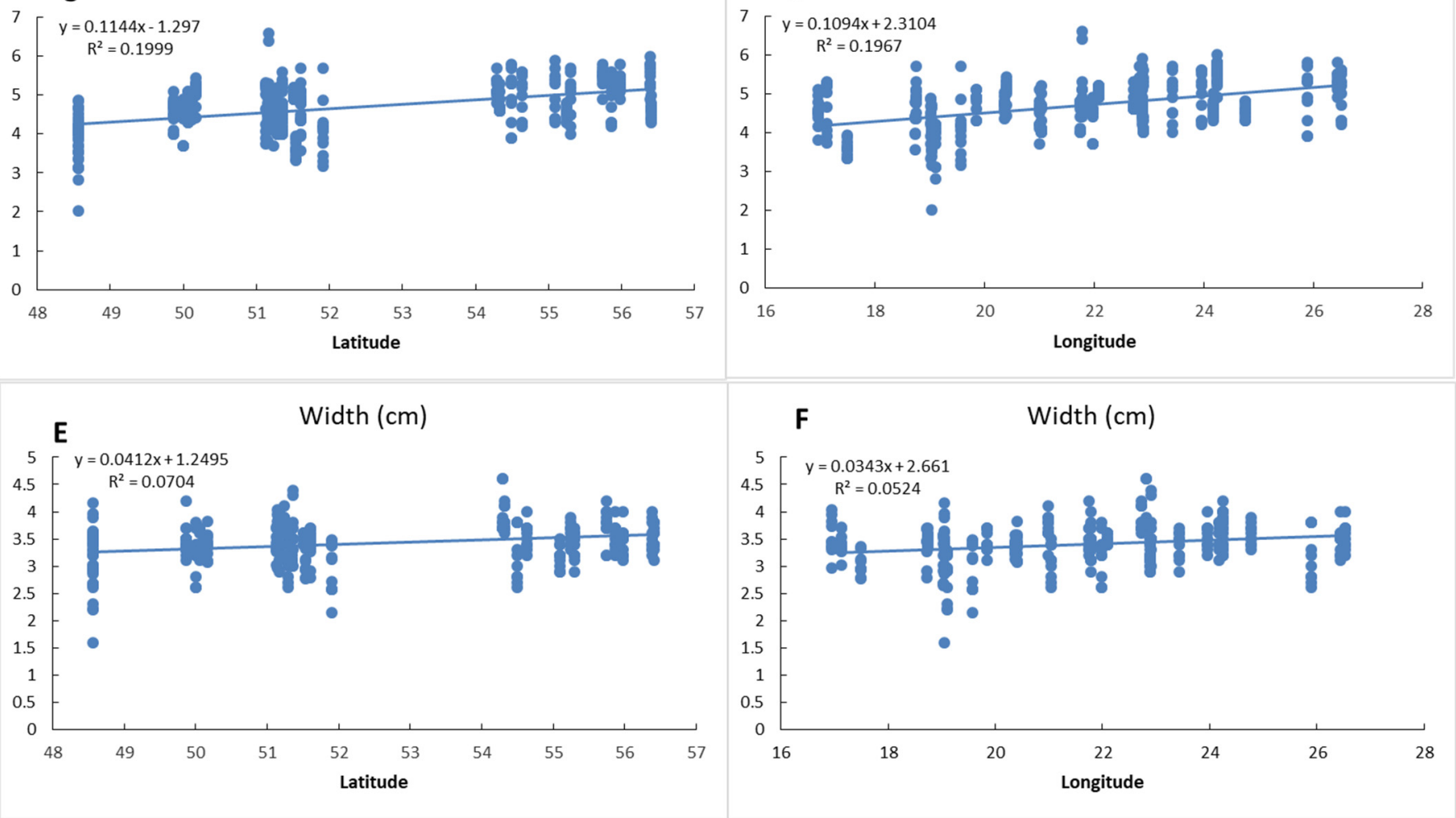

Figure 4. Correlation of fruit size of Echinocystis lobata with geographical position. The fruits from natural habitats $(N=339)$ from Central Europe locations $(n=34)$ for height level of $1.01-1.5 \mathrm{~m}$. $(\mathbf{A}, \mathbf{B})$ - fresh weight of fruits, $(\mathbf{C}, \mathbf{D})$ - length of fresh fruits, $(\mathbf{E}, \mathbf{F})$ - - width of fresh fruits $(p<0.005$, F test). 


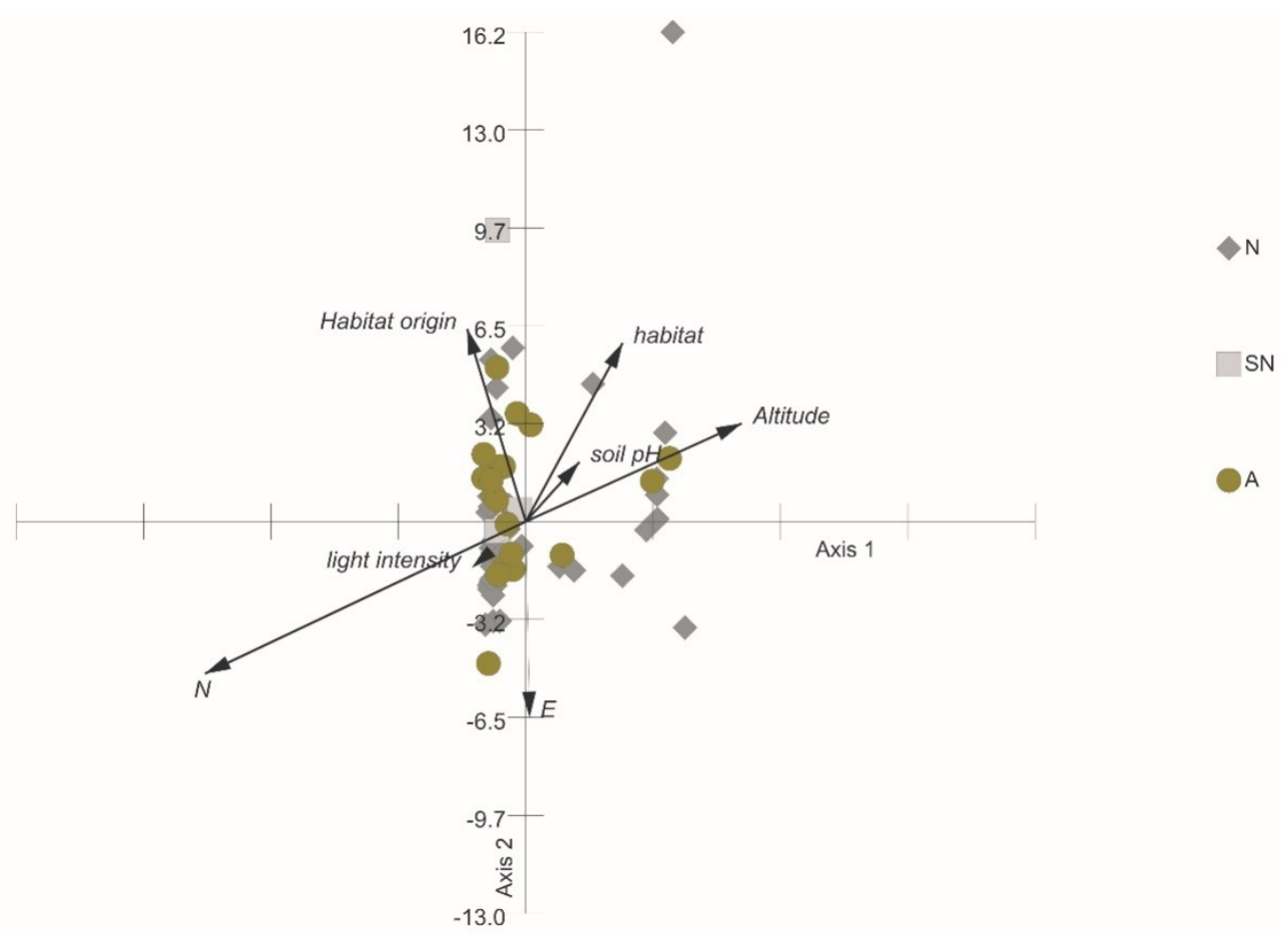

Figure 5. The CCA diagram for Echinocystis lobata fruit parameters per locality with relation to environmental variables. N, E-geographical position; N, SN, A-habitat origin-natural, seminatural, and anthropogenic.

\section{Discussion}

In Central and Eastern Europe, populations of Ecinocystis lobata can occupy anthropogenic, semi-natural, and natural habitats. We showed that the fruit size and number of seeds are dependent on the origin and type of habitats. The fruits of $E$. lobata were bigger and heavier in natural habitats than in semi-natural and anthropogenic ones, and the number of seeds was the greatest in semi-natural habitats. On the other hand, the origin of habitat did not affect the number of undeveloped seeds (Table 1). Considering the types of habitats, the greatest fruit length and weight were observed in mires, bogs, and fens (habitat $\mathrm{D}$ in the group of natural habitats), while the greatest number of seeds was found on banks and shores of inland surface waters (habitat $C$ in the group of natural habitats) (Table 2). Our results suggest that the size of the fruit and the number of seeds of E. lobata are influenced by the water availability in the habitat. Many annual climbers of Cucurbitaceae show high water requirements because they have a relatively small root system and produce an extremely large above-ground mass of leaves as well as fruits [50]. E. lobata has shallow roots (4-15 cm long and about $1 \mathrm{~mm}$ wide) and long climbing to trailing stems (5-12 $\mathrm{m}$ long and 3-5 mm thick) with numerous leaves and coiled tendrils [37]. The pepo, a simple fleshy fruit typical of Cucurbitaceae [51], contains a lot of water. For example, fruits of cucurbit crops Cucumis sativus L., Lagenaria siceraria (Molina) Standl, and Luffa acutangula (L.) Roxb. consist of more than $95 \%$ water [12]. The pepo of E. lobata is also very moist, but dries to tan after seeds are expelled [37]. The formation of the pepo requires adequate access to water and wet habitats may enhance the production of big-sized fruits with a greater number of seeds by providing plants with more water [52]. Experiments conducted on Cucumis sativus showed that, during the reproductive stage, the plants were more sensitive to water deficit resulting in a decrease in fruit weight [53]. In cucurbit crops (Cucurbita, Cucumis, and Citrullus), the size of the fruit decreases not only because of reduced water supply, but also because of increased plant density and a greater number of fruits per plant [54]. Moreover, light availability and soil fertility could be responsible for fruit weight and seed production in members of Cucurbitaceae, as evidenced in Sicyos angulatus [55]. 
Echinocystis lobata grows fast and is adapted to moist and nutrient-rich habitats [35]. It is considered one of the most abundant invasive alien plants spreading in natural riparian habitats [56], whereas it is a rare species in urban woodlands [57]. In riparian habitats, where the soils are wet and fertile, E. lobata can achieve high seed viability and germinability and its rate of seedling recruitment is higher than in dry and nutrient-poor soils [34,58,59]. Similarly, seed germination of $S$. angulatus occurs more frequently in humid and irrigated sites [60].

The fruits of E. lobata can be developed at different heights because of the climbing strategy. In some climbing plants, the use of external support modifies biomass allocation and stimulates growth, whereas in other species, it may negatively affect growth, indicating a cost associated with climbing $[1,61]$. Moreover, the non-climbing plants that provide mechanical support for the climbing plants are also competitors for light, water, and nutrients [1,62]. In the case of E. lobata, the fruit production increases with plant size, but is limited by the number of nodes [35]. Our results suggest that the length and the weight of the fruits, as well as the number and the weight of the seeds, can be affected by the height at which the plant develops the fruits. Considering habitat types, in mires, bogs, and fens (habitat type D), the fruit length and weight and the number of seeds decreased with the increasing height (Table 6, Table 8, and Table 9). The lowest number of seeds observed in forest habitats at a height of 3.51-4.00 m, as well as in habitat complexes, particularly at a height of 3.01-3.50 m (Table 9), might be caused by an elevated temperature. The extremely detrimental effects of heat stress on seed yield have been documented in numerous plant species and repeatedly reported by many authors [63-65]. The lowest number of seeds in fruits of E. lobata growing at a substantial height might be also related to fruit set order. However, the youngest fruits are not always located at the highest shoots. The development of fruits and seeds depends mainly on carbohydrates synthesised in and transported from vegetative tissues of leaves and stems. Thus, there is a competition between reproductive organs located on the same mother plant [66]. Studies on cucurbit crops (i.e., Cucumis melo, C. sativus, Citrullus lanatus, and Cucurbita pepo) have proved the decrease in the number of seeds $[67,68]$ and their quality [69] in fruits that originate later. Moreover, in cucurbit crops, the fruit already developing on the plant hinders successful fruit set in younger flowers, especially those on the same branch or stem [54]. Interestingly, in Cucumis sativus, the higher the fruit position on the mother plant and the closer the ovule to the stem (in the peduncular fruit segment), the slower the maturation of the seeds and the lower the vigour when harvested early [66]. In cucurbit crops, the development of fruits and seeds is impacted by various genetic and environmental factors [54]. Further studies are needed for better understanding of the variability in fruit size and seed production in populations of E. lobata. It seems important to check the frequency and efficiency of insect pollination and self-pollination in flowers located at different heights. It has been shown that, if the amount of pollen is insufficient, or conditions are not suitable for pollen tube growth, a misshapen fruit occurs in cucurbit crops [54].

In this study, we found a positive correlation between the weight and the length of the fruits of E. lobata (Table 11). A similar correlation was evidenced for other species of Cucurbitaceae such as Trichosanthes dioica Roxb. [70], Cucumis melo L. [71], and Citrullus lanatus (Thunb.) Mansf. [72]. Moreover, we found a positive correlation between the fruit size and the number of seeds of E. lobata (Table 11), as observed in cucurbit crops such as Cucumis satious [73] and Cucurbita pepo L. [74]. In maturing fruits of E. lobata, turgor pressure provides their rupture and autochorous seed dispersal [34]. Seeds fall from the fruits irregularly; most seeds are released just after the opening of the fruit, while the rest are released after the drying and decaying of the fruit $[35,75]$. The fruit usually contains four seeds $[32,35,75]$. The seeds of E. lobata are large and smooth, with no special structures for long-distance dispersal. According to Dylewski et al. [75], the mean seed mass is $0.33 \mathrm{~g}$, the mean length of the seed is $17 \mathrm{~mm}$, and the mean width of the seed is $8 \mathrm{~mm}$. Similar results of seed size measurements were obtained by Golivets [76]. Interestingly, Dylewski et al. [75] hypothesised that E. lobata produces more seeds (up to six) and some 
of the seeds are trapped in the fruit as a way of physical defence against rodents and birds that feed on the seeds. Our results suggest that the defence against seed-eating animals might be the strongest in semi-natural habitats, as well as on banks and shores of inland surface waters, as E. lobata produces the highest number of seeds in these habitats. In general, seed predation is an important factor reducing seed availability and could be an evolutionary driver [77-81]. On the other hand, rodents and birds can serve as vectors of seed dispersal [32,75], and mutualism involving animal-mediated seed dispersal facilitates plant invasions [82]. The formation of fruits at different heights may lead to more successful seed dispersal by animals and wind. The seeds of fruits developed at low heights can be dispersed by rodents, whereas the seeds of fruits located higher can be dispersed by birds. The wind can also enhance seed dispersal by moving the branches of trees and shrubs on which the fruiting shoots of E. lobata are supported. Furthermore, the seeds of E. lobata can also be dispersed by water during floods along the riverbanks [29]. The pattern of spread of E. lobata along river valleys has been confirmed in many regions of Europe [28,30,31,40,45,83]. Compared with other invasive vines of the Cucurbitaceae (e.g., Coccinia grandis and Thladiantha dubia), E. lobata is not able to propagate vegetatively. However, the seeds of E. lobata can be dispersed by autochory, hydrochory, and zoochory $[29,32,35,75]$. Such a diversity of seed dispersal favours the rapid spread and invasion of E. lobata in Central and Eastern Europe [29].

Seed weight is a critical trait of a plant's life history; it influences many aspects of a species' regeneration strategy, including seedling survival rate. When considering variation between species, seed size exhibits a striking global pattern, declining 2-3 orders of magnitude going to the north [84]. Several theories have been proposed to explain this latitudinal gradient, involving light conditions, dispersal agents, life form, and length of the growing season [85]. Similarly, global-scale research in fleshy fruit colour and size has documented geographic patterns between species traits and observed the negative correlation between size and latitude progressing towards the north [81]. On a smaller scale, with increasing elevation in the mountains, the pattern of seed size is not so obvious, and the relationship between elevation and seed mass may be positive [86]. Additionally, the seed size within the same species across an elevation is not always consistent with the general global pattern, and the response to elevation change seems to be the plant's trait [87-89]. The seed size shows a considerable degree of phenotypic plasticity in response to environmental conditions [90]. The variation in seed size has been investigated in many cucurbit crops [91]. For example, the geographical variability of seed length, width, and thickness was observed in Cucurbita pepo by Paris and Nerson [92]. In our research, we did not find a geographic pattern in seed size within species; the observed variability is included in the phenotypic plasticity of the species. However, the geographic pattern was revealed for fruits. We noticed significantly larger fruit at locations in the northeast in our dataset. We assumed that, at higher latitudes and under cooler temperatures, the seeds ripen more slowly, and the longer period is exploited for fruit growth. There are numerous ecophysiological traits strongly associated with plant invasions such as high leaf construction cost, specific leaf area, net $\mathrm{CO}_{2}$ assimilation, high photosynthesis capacity, net photosynthesis, rapid and substantial allocation to root mass, heteroblasty, strong $\mathrm{N}$ fixation ability, and seed dry weight [93]. Unfortunately, ecophysiological aspects of E. lobata invasion have not been intensively studied so far. The experiment performed in the riparian zone of the river Krka in Slovenia indicated that E. lobata had significantly higher specific leaf area and lower leaf tissue density compared with Salix caprea L. and S. fragilis L. (which provided mechanical support for E. lobata shoots), altering the radiation environment and the amount of photosynthetic pigments [9]. Fruit and seed traits can be responsible for the invasiveness of alien plant species because they are related to dispersal, germination, and fitness [94]. According to Golivets [76] and Dylewski et al. [75], the seeds of E. lobata are very variable in their weight, length, width, and coloration. However, the effect of seed traits (including ecophysiological traits) on germination capacity and seedling survival in various habitats and geographic regions (in comparison with the native 
range) should be tested in the future to reveal their involvement in the invasiveness of E. lobata.

\section{Conclusions}

In Central and Eastern Europe, Echinocystis lobata showed great variability in the size of fruits and seeds depending on the type and origin of the habitat as well as depending on the height at which the fruits were formed as a result of climbing. High water availability in the soil seems to have a positive effect on fruit size as the largest fruits were found in natural riparian habitats. The number of seeds was positively correlated with the width, length, and fresh weight of the fruit. The distribution of fruits at different heights may contribute to better dispersal of seeds by animals and wind, and may also better protect the seeds from being eaten by granivorous animals. The defence against granivorous animals is expected to be stronger in semi-natural habitats, as well as on banks and shores of inland surface waters, as the plant produced the highest number of seeds per fruit in these habitats. The invasiveness of E. lobata in various habitats and regions in Europe requires further research. It seems particularly important to demonstrate the seed germination capacity and seedling survival depending on the habitat conditions, as well as to indicate seed traits strongly involved in the invasiveness of the species.

Author Contributions: Conceptualization: K.K.-G., A.P., A.S.-S. and Z.G.; methodology: K.K.-G., A.P., A.S.-S. and Z.G.; statistical analysis: K.K.-G. and A.S.-S.; investigation: K.K.-G., A.P., B.B.-K., A.B., Z.D., Z.G., M.K., Ł.M., J.M., K.M., M.P., M.R., A.R., E.S., T.W. and A.S.-S.; resources: K.K.-G., A.P., B.B.-K., A.B., Z.D., Z.G, M.K., Ł.M., J.M., K.M., M.P., M.R., A.R., E.S., T.W. and A.S.-S.; data curation: K.K.-G., A.P. and A.S.-S.; writing-original draft preparation: K.K.-G., A.P. and A.S.-S.; writing-review and editing: K.K.-G., A.P. and A.S.-S.; supervision: K.K.-G., A.P. and A.S.-S.; project administration: K.K.-G., A.P. and A.S.-S. All authors have read and agreed to the published version of the manuscript.

Funding: This research received no external funding.

Institutional Review Board Statement: Not applicable.

Informed Consent Statement: Not applicable.

Data Availability Statement: Not applicable.

Acknowledgments: We thank the Reviewers and Editor for their valuable comments, which helped improve our manuscript. We also thank the Committee for Mapping the Flora of Europe (University of Helsinki, Finland) for allowing us to use the base map.

Conflicts of Interest: The authors declare no conflict of interest.

\section{Appendix A}

Table A1. Characteristics of study sites located in Latvia (LV), Lithuania (LT), Poland (PL), and Slovakia (SK).

\begin{tabular}{|c|c|c|c|c|c|c|c|c|c|c|}
\hline \multirow{2}{*}{$\begin{array}{l}\text { Country } \\
\text { Code }\end{array}$} & \multirow{2}{*}{ Study Site } & \multicolumn{2}{|c|}{ GPS Coordinates } & \multirow{2}{*}{$\begin{array}{l}\text { Altitude } \\
\text { (m a.s.1.) }\end{array}$} & \multicolumn{3}{|c|}{ Habitat } & \multirow{2}{*}{$\begin{array}{c}\text { Plant } \\
\text { Cover }\end{array}$} & \multirow{2}{*}{$\begin{array}{l}\text { Soil } \\
\text { pH }\end{array}$} & \multirow{2}{*}{$\begin{array}{c}\text { Light } \\
\text { Availability ** }\end{array}$} \\
\hline & & Latitude & Longitude & & Code & Type & Origin & & & \\
\hline LV & $\begin{array}{c}\text { Daugavpils, right } \\
\text { bank of the } \\
\text { Daugava river }\end{array}$ & $\frac{\mathrm{N}}{55^{\circ} 51^{\prime} 53.4^{\prime \prime}}$ & $\begin{array}{c}\mathrm{E} \\
26^{\circ} 31^{\prime} 13.9^{\prime \prime}\end{array}$ & 295 & F9.121 & $\begin{array}{l}\text { Almond } \\
\text { willow-osier } \\
\text { scrub }\end{array}$ & $\mathrm{N}$ & $\begin{array}{c}\text { B30\% } \\
\text { C-90\% }\end{array}$ & 7.1 & 3 \\
\hline LV & $\begin{array}{c}\text { Daugavpils } \\
\text { district, Tilti } \\
\text { village, bank of } \\
\text { the Tūkšna river }\end{array}$ & $\frac{\mathrm{N}}{55^{\circ} 58^{\prime} 24.3^{\prime \prime}}$ & $\begin{array}{c}\mathrm{E} \\
26^{\circ} 26^{\prime} 27.3^{\prime \prime}\end{array}$ & 289 & G1.11 & $\begin{array}{c}\text { Riverine Salix } \\
\text { woodland }\end{array}$ & $\mathrm{N}$ & $\begin{array}{l}\text { A- } 20 \% \\
\text { B- } 40 \% \\
\text { C- } 80 \%\end{array}$ & 6.9 & 2 \\
\hline $\mathrm{LV}$ & $\begin{array}{c}\text { Jekabpils, left } \\
\text { bank of the } \\
\text { Daugava River }\end{array}$ & $\begin{array}{c}\mathrm{N} \\
56^{\circ} 29^{\prime} 43.0^{\prime \prime}\end{array}$ & $\underset{25^{\circ} 53^{\prime} 52.1^{\prime \prime}}{\mathrm{E}}$ & 296 & F9.121 & $\begin{array}{l}\text { Almond } \\
\text { willow-osier } \\
\text { scrub }\end{array}$ & $\mathrm{N}$ & $\begin{array}{l}\text { B- } 40 \% \\
\text { C-90\% }\end{array}$ & 7.1 & 3 \\
\hline
\end{tabular}


Table A1. Cont.

\begin{tabular}{|c|c|c|c|c|c|c|c|c|c|c|}
\hline \multirow{2}{*}{$\begin{array}{l}\text { Country } \\
\text { Code }\end{array}$} & \multirow{2}{*}{ Study Site } & \multicolumn{2}{|c|}{ GPS Coordinates } & \multirow{2}{*}{$\begin{array}{l}\text { Altitude } \\
\text { (m a.s.l.) }\end{array}$} & \multicolumn{3}{|c|}{ Habitat } & \multirow{2}{*}{$\begin{array}{c}\text { Plant } \\
\text { Cover * }\end{array}$} & \multirow{2}{*}{$\begin{array}{c}\text { Soil } \\
\text { pH }\end{array}$} & \multirow{2}{*}{$\begin{array}{c}\text { Light } \\
\text { Availability ** }\end{array}$} \\
\hline & & Latitude & Longitude & & Code & Type & Origin & & & \\
\hline $\mathrm{LV}$ & $\begin{array}{l}\text { Bauska, right } \\
\text { bank of the } \\
\text { Memele river }\end{array}$ & $\underset{56^{\circ} 24^{\prime} 15.2^{\prime \prime}}{\mathrm{N}}$ & $\begin{array}{c}\mathrm{E} \\
24^{\circ} 10^{\prime} 29.4^{\prime \prime}\end{array}$ & 40 & G1.11 & $\begin{array}{l}\text { Riverine Salix } \\
\text { woodland }\end{array}$ & $\mathrm{N}$ & $\begin{array}{l}\text { A-20\% } \\
\text { B-20\% } \\
\text { C-95\% }\end{array}$ & 7.3 & 3 \\
\hline LV & $\begin{array}{l}\text { Bauska district, } \\
\text { Arce village, right } \\
\text { bank of the } \\
\text { Mūša river }\end{array}$ & $\begin{array}{c}\mathrm{N} \\
56^{\circ} 23^{\prime} 3.7^{\prime \prime}\end{array}$ & $\begin{array}{c}\mathrm{E} \\
24^{\circ} 15^{\prime} 03.6^{\prime \prime}\end{array}$ & 35 & C 3.21 & $\begin{array}{l}\text { Common reed } \\
\text { (Phragmites) } \\
\text { beds }\end{array}$ & $\mathrm{N}$ & C- $-100 \%$ & 7.1 & 4 \\
\hline LT & $\begin{array}{l}\text { Prienai district, } \\
\text { Prienai, right } \\
\text { bank of the } \\
\text { Nemunas River }\end{array}$ & $\underset{54^{\circ} 37^{\prime} 55.6^{\prime \prime}}{\mathrm{N}}$ & $\begin{array}{c}\mathrm{E} \\
23^{\circ} 57^{\prime} 16.5^{\prime \prime}\end{array}$ & 51 & G1.11 & $\begin{array}{l}\text { Riverine Salix } \\
\text { woodland }\end{array}$ & $\mathrm{N}$ & $\begin{array}{l}\text { A-20\% } \\
\text { B-30\% } \\
\text { C- } 95 \%\end{array}$ & 7.1 & 3 \\
\hline LT & $\begin{array}{l}\text { Trakai district, } \\
\text { Trakai }\end{array}$ & $\begin{array}{c}\mathrm{N} \\
54^{\circ} 38^{\prime} 50.9^{\prime \prime}\end{array}$ & $\begin{array}{c}\mathrm{E} \\
24^{\circ} 55^{\prime} 04.3^{\prime \prime}\end{array}$ & 165 & $\mathrm{H} 3.1 \mathrm{C}$ & $\begin{array}{l}\text { Disused } \\
\text { siliceous } \\
\text { quarries }\end{array}$ & A & C- $60 \%$ & 7.6 & 5 \\
\hline LT & $\begin{array}{l}\text { Ukmergèdistr, } \\
\text { Ukmergè, left } \\
\text { bank of the } \\
\text { Šventoji River }\end{array}$ & $\underset{55^{\circ} 14^{\prime} 38.1^{\prime \prime}}{\mathrm{N}}$ & $\begin{array}{c}\mathrm{E} \\
24^{\circ} 45^{\prime} 50.5^{\prime \prime}\end{array}$ & 56 & F9.121 & $\begin{array}{l}\text { Almond } \\
\text { willow-osier } \\
\text { scrub }\end{array}$ & $\mathrm{N}$ & $\begin{array}{l}\text { B- } 60 \% \\
\text { C- } 80 \%\end{array}$ & 6.7 & 3 \\
\hline LT & $\begin{array}{l}\text { Panevèžys district, } \\
\text { Berčiūnai, right } \\
\text { bank of the } \\
\text { Nevėžis River }\end{array}$ & $\underset{55^{\circ} 44^{\prime} 19.3^{\prime \prime}}{\mathrm{N}}$ & $\begin{array}{c}\mathrm{E} \\
24^{\circ} 14^{\prime} 24.0^{\prime \prime}\end{array}$ & 41 & F9.121 & $\begin{array}{l}\text { Almond } \\
\text { willow-osier } \\
\text { scrub }\end{array}$ & $\mathrm{N}$ & $\begin{array}{l}\text { A- } 10 \% \\
\text { B- } 50 \% \\
\text { C- } 90 \%\end{array}$ & 6.9 & 3 \\
\hline LT & $\begin{array}{l}\text { Šiauliai district, } \\
\text { Norkūnai village }\end{array}$ & $\begin{array}{c}\mathrm{N} \\
56^{\circ} 01^{\prime} 12.9^{\prime \prime}\end{array}$ & $\begin{array}{c}\mathrm{E} \\
23^{\circ} 16^{\prime} 55.6^{\prime \prime}\end{array}$ & 94 & $\mathrm{~J} 2.7$ & $\begin{array}{c}\text { Rural } \\
\text { construction } \\
\text { and } \\
\text { demolition } \\
\text { sites }\end{array}$ & $\mathrm{A}$ & $\begin{array}{l}\text { B- } 10 \% \\
\text { C-70\% }\end{array}$ & 7.3 & 4 \\
\hline LT & $\begin{array}{l}\text { Raseiniai district, } \\
\text { Vandžiai village, } \\
\text { left bank of the } \\
\text { Dubysa River }\end{array}$ & $\underset{55^{\circ} 18^{\prime} 01.9^{\prime \prime}}{\mathrm{N}}$ & $\begin{array}{c}\mathrm{E} \\
23^{\circ} 25^{\prime} 46.0^{\prime \prime}\end{array}$ & 40 & F9.121 & $\begin{array}{c}\text { Almond } \\
\text { willow-osier } \\
\text { scrub }\end{array}$ & $\mathrm{N}$ & $\begin{array}{l}\text { B- } 40 \% \\
\text { C-70\% }\end{array}$ & 6.8 & 4 \\
\hline LT & $\begin{array}{l}\text { Jurbarkas district, } \\
\text { Skirsnemune, } \\
\text { right bank of the } \\
\text { Nemunas River }\end{array}$ & $\begin{array}{c}\mathrm{N} \\
55^{\circ} 05^{\prime} 25.9^{\prime \prime}\end{array}$ & $\begin{array}{c}\mathrm{E} \\
22^{\circ} 52^{\prime} 50.16^{\prime \prime}\end{array}$ & 13 & F9.121 & $\begin{array}{c}\text { Almond } \\
\text { willow-osier } \\
\text { scrub }\end{array}$ & $\mathrm{N}$ & $\begin{array}{l}\text { B- } 50 \% \\
\text { C- } 80 \%\end{array}$ & 6.8 & 4 \\
\hline PL & Filipów Pierwszy & $\begin{array}{c}\mathrm{N} \\
54^{\circ} 17^{\prime} 42^{\prime \prime}\end{array}$ & $\begin{array}{c}\mathrm{E} \\
22^{\circ} 49^{\prime} 22^{\prime \prime}\end{array}$ & 166 & D5.2121 & $\begin{array}{l}\text { Slender tufted } \\
\text { sedge beds } \\
\text { near } \\
\text { sub-boreal } \\
\text { swamp alder } \\
\text { woods } \\
\text { (G1.4114) } \\
\end{array}$ & $\mathrm{N}$ & C- $-95 \%$ & 6.9 & 4 \\
\hline PL & Bakałarzewo & $\begin{array}{c}\mathrm{N} \\
54^{\circ} 19^{\prime} 29^{\prime \prime}\end{array}$ & $\underset{22^{\circ} 43^{\prime} 00^{\prime \prime}}{ }$ & 154 & G1.1112 & $\begin{array}{c}\text { Eastern } \\
\text { European } \\
\text { poplar-willow } \\
\text { forests }\end{array}$ & $\mathrm{N}$ & $\begin{array}{l}\text { A- } 50 \% \\
\text { B-75\% } \\
\text { C-75\% }\end{array}$ & 7.6 & 2 \\
\hline PL & $\begin{array}{c}\text { Grodzisk } \\
\text { Wielkopolski }\end{array}$ & $\stackrel{\mathrm{N}}{\mathrm{N} 2^{\circ} 13^{\prime} 10.92^{\prime \prime}}$ & $\begin{array}{c}E \\
16^{\circ} 22^{\prime} 36.06^{\prime \prime}\end{array}$ & 80 & $\mathrm{I} 2.2$ & $\begin{array}{l}\text { Small-scale } \\
\text { ornamental } \\
\text { and domestic } \\
\text { garden areas }\end{array}$ & A & C- $50 \%$ & 7.1 & 5 \\
\hline PL & Kamieniec & $\underset{52^{\circ} 09^{\prime} 53.46^{\prime \prime}}{\mathrm{N}}$ & $\begin{array}{c}\mathrm{E} \\
16^{\circ} 28^{\prime} 20.52^{\prime \prime}\end{array}$ & 68 & C3.63 & $\begin{array}{l}\text { Unvegetated } \\
\text { river mud } \\
\text { banks }\end{array}$ & $\mathrm{N}$ & C- $80 \%$ & 7.6 & 4 \\
\hline PL & Goździchowo & $\begin{array}{c}\mathrm{N} \\
52^{\circ} 09^{\prime} 22.14^{\prime \prime}\end{array}$ & $\begin{array}{c}\mathrm{E} \\
16^{\circ} 28^{\prime} 28.08^{\prime \prime}\end{array}$ & 53 & C 3.63 & $\begin{array}{l}\text { Unvegetated } \\
\text { river mud } \\
\text { banks }\end{array}$ & $\mathrm{N}$ & C-70\% & 7.6 & 4 \\
\hline
\end{tabular}


Table A1. Cont.

\begin{tabular}{|c|c|c|c|c|c|c|c|c|c|c|}
\hline \multirow{2}{*}{$\begin{array}{l}\text { Country } \\
\text { Code }\end{array}$} & \multirow{2}{*}{ Study Site } & \multicolumn{2}{|c|}{ GPS Coordinates } & \multirow{2}{*}{$\begin{array}{l}\text { Altitude } \\
\text { (m a.s.l.) }\end{array}$} & \multicolumn{3}{|c|}{ Habitat } & \multirow{2}{*}{$\begin{array}{l}\text { Plant } \\
\text { Cover* }\end{array}$} & \multirow{2}{*}{$\begin{array}{l}\text { Soil } \\
\text { pH }\end{array}$} & \multirow{2}{*}{$\begin{array}{c}\text { Light } \\
\text { Availability ** }\end{array}$} \\
\hline & & Latitude & Longitude & & Code & Type & Origin & & & \\
\hline PL & Bęczkowice & $\stackrel{\mathrm{N}}{\mathrm{N}}$ & $\begin{array}{c}E \\
19^{\circ} 42^{\prime} 37.41^{\prime \prime}\end{array}$ & 208 & E3.4 & $\begin{array}{l}\text { Moist or wet } \\
\text { eutrophic and } \\
\text { mesotrophic } \\
\text { grassland }\end{array}$ & SN & $\begin{array}{c}\text { A-5\% } \\
\text { B- } 5 \% \\
\text { C- } 90 \%\end{array}$ & 6.4 & 4 \\
\hline PL & Mileszki & $\begin{array}{c}\mathrm{N} \\
5^{\circ} 46^{\prime} 15.58^{\prime \prime}\end{array}$ & $\begin{array}{c}E \\
19^{\circ} 34^{\prime} 26.79^{\prime \prime}\end{array}$ & 240 & $\mathrm{I} 2.2$ & $\begin{array}{c}\text { Small-scale } \\
\text { ornamental } \\
\text { and domestic } \\
\text { garden areas }\end{array}$ & A & $\begin{array}{l}\text { A- } 10 \% \\
\text { B- } 90 \% \\
\text { C-70\% }\end{array}$ & 5.4 & 3 \\
\hline PL & Ługi & $\begin{array}{c}\mathrm{N} \\
5_{51^{\circ} 46^{\prime} 15.58^{\prime \prime}}\end{array}$ & $\begin{array}{c}E \\
19^{\circ} 34^{\prime} 26.79^{\prime \prime}\end{array}$ & 177 & $\mathrm{~J} 4.2$ & $\begin{array}{c}\text { Road } \\
\text { networks }\end{array}$ & $\mathrm{A}$ & $\begin{array}{l}\text { A- } 10 \% \\
\text { B- } 10 \% \\
\text { C- } 95 \%\end{array}$ & 7.3 & 4 \\
\hline PL & Nowa Brzeźnica & $\begin{array}{c}\mathrm{E} \\
19^{\circ} 10^{\prime} 52.46^{\prime \prime}\end{array}$ & $\begin{array}{c}\mathrm{E} \\
19^{\circ} 10^{\prime} 52.46^{\prime \prime}\end{array}$ & 203 & G1.1 & $\begin{array}{l}\text { Riparian and } \\
\text { gallery } \\
\text { woodland }\end{array}$ & $\mathrm{N}$ & $\begin{array}{l}\text { A- } 40 \% \\
\text { B-70\% } \\
\text { C-70\% }\end{array}$ & 6.3 & 2 \\
\hline PL & Sieradz 1 & $\underset{51^{\circ} 35^{\prime} 58.29^{\prime \prime}}{\mathrm{N}}$ & $\begin{array}{c}\mathrm{E} \\
18^{\circ} 44^{\prime} 35.71^{\prime \prime}\end{array}$ & 129 & G1.1 & $\begin{array}{l}\text { Riparian and } \\
\text { gallery } \\
\text { woodland }\end{array}$ & $\mathrm{N}$ & $\begin{array}{l}\mathrm{A}-5 \% \\
\mathrm{~B}-80 \% \\
\mathrm{C}-90 \%\end{array}$ & 6.1 & 3 \\
\hline PL & Sieradz 2 & $\begin{array}{c}\mathrm{N} \\
51^{\circ} 36^{\prime} 27.10^{\prime \prime}\end{array}$ & $\begin{array}{c}\mathrm{E} \\
18^{\circ} 43^{\prime} 47,42^{\prime \prime}\end{array}$ & 136 & G1.1 & $\begin{array}{l}\text { Riparian and } \\
\text { gallery } \\
\text { woodland }\end{array}$ & $\mathrm{N}$ & $\begin{array}{c}\mathrm{A}-5 \% \\
\mathrm{~B}-80 \% \\
\mathrm{C}-90 \%\end{array}$ & 6.2 & 3 \\
\hline PL & Smolice & $\underset{51^{\circ} 54^{\prime} 17.24^{\prime \prime}}{\mathrm{N}}$ & $\begin{array}{c}E \\
19^{\circ} 34^{\prime} 16.68^{\prime \prime}\end{array}$ & 153 & G1.1 & $\begin{array}{l}\text { Riparian and } \\
\text { gallery } \\
\text { woodland }\end{array}$ & $\mathrm{N}$ & $\begin{array}{l}\text { A- } 40 \% \\
\text { B- } 30 \% \\
\text { C- } 80 \%\end{array}$ & 7.2 & 3 \\
\hline PL & $\begin{array}{c}\text { Smolice, } \\
\text { Wczasowa Street }\end{array}$ & $\underset{51^{\circ} 54^{\prime} 05.62^{\prime \prime}}{\mathrm{N}}$ & $\frac{E}{19^{\circ} 34^{\prime} 54.65^{\prime \prime}}$ & 158 & $\mathrm{~J} 2.5$ & $\begin{array}{l}\text { Constructed } \\
\text { boundaries }\end{array}$ & $\mathrm{A}$ & $\begin{array}{l}\text { A- } 10 \% \\
\text { B-20\% } \\
\text { C- } 20 \%\end{array}$ & 6.4 & 5 \\
\hline PL & Witkowice & $\begin{array}{c}\mathrm{N} \\
5_{51^{\circ} 46^{\prime} 21.94^{\prime \prime}}\end{array}$ & $\begin{array}{c}E \\
19^{\circ} 44^{\prime} 53.78^{\prime \prime}\end{array}$ & 216 & $\mathrm{~J} 2.5$ & $\begin{array}{l}\text { Constructed } \\
\text { boundaries }\end{array}$ & A & $\begin{array}{c}\text { A- } 10 \% \\
\text { B- } 5 \% \\
\text { C-60\% }\end{array}$ & 6.5 & 4 \\
\hline PL & Łęczna & $\begin{array}{c}\mathrm{N} \\
51^{\circ} 21^{\prime} 23^{\prime \prime}\end{array}$ & $\begin{array}{c}\mathrm{E} \\
22^{\circ} 54^{\prime} 18^{\prime \prime}\end{array}$ & 150 & G1.11 & $\begin{array}{l}\text { Riverine Salix } \\
\text { woodland }\end{array}$ & $\mathrm{N}$ & $\begin{array}{c}\text { A-40\% } \\
\text { B-30\% } \\
\text { C-100\% }\end{array}$ & 6.5 & 3 \\
\hline PL & Wólka & $\begin{array}{c}\mathrm{N} \\
51^{\circ} 21^{\prime} 03^{\prime \prime}\end{array}$ & $\begin{array}{c}\mathrm{E} \\
22^{\circ} 52^{\prime} 60^{\prime \prime}\end{array}$ & 177 & G1.11 & $\begin{array}{l}\text { Riverine Salix } \\
\text { woodland }\end{array}$ & $\mathrm{N}$ & $\begin{array}{l}\text { A- } 60 \% \\
\text { B-30\% } \\
\text { C- } 90 \%\end{array}$ & 6.5 & 2 \\
\hline PL & Łysaków & $\begin{array}{c}\mathrm{N} \\
51^{\circ} 31^{\prime} 50^{\prime \prime}\end{array}$ & $\begin{array}{c}E \\
22^{\circ} 38^{\prime} 60^{\prime \prime}\end{array}$ & 160 & X25 & $\begin{array}{l}\text { Domestic } \\
\text { gardens of } \\
\text { villages and } \\
\text { urban } \\
\text { peripheries }\end{array}$ & A & $\begin{array}{l}\text { B-70\% } \\
\text { C- } 90 \%\end{array}$ & 6.0 & 3 \\
\hline PL & Kępa Solecka & $\begin{array}{c}\mathrm{N} \\
51^{\circ} 10^{\prime} 9^{\prime \prime}\end{array}$ & $\begin{array}{c}\mathrm{E} \\
21^{\circ} 46^{\prime} 60^{\prime \prime}\end{array}$ & 123 & G1.22 & $\begin{array}{c}\text { Mixed } \\
\text { oak-elm-ash } \\
\text { woodland of } \\
\text { great rivers }\end{array}$ & $\mathrm{N}$ & $\begin{array}{c}\text { A- } 20 \% \\
\text { B- } 40 \% \\
\text { C- } 100 \%\end{array}$ & 6.5 & 3 \\
\hline PL & $\begin{array}{c}\text { Zastów } \\
\text { Polanowski }\end{array}$ & $\frac{\mathrm{N}}{51^{\circ} 30^{\prime} 31^{\prime \prime}}$ & $\begin{array}{c}\mathrm{E} \\
22^{\circ} 05^{\prime} 09^{\prime \prime}\end{array}$ & 119 & G1.11 & $\begin{array}{l}\text { Riverine Salix } \\
\text { woodland }\end{array}$ & $\mathrm{N}$ & $\begin{array}{l}\text { A-20\% } \\
\text { B-30\% } \\
\text { C- } 90 \%\end{array}$ & 6.5 & 3 \\
\hline PL & Niekłań 1 & $\underset{51^{\circ} 16^{\prime} 38.43^{\prime \prime}}{\mathrm{N}}$ & $\frac{E}{20^{\circ} 61^{\prime} 79.85^{\prime \prime}}$ & 279 & E5.14 & $\begin{array}{c}\text { Weed } \\
\text { communities } \\
\text { of recently } \\
\text { abandoned } \\
\text { extractive } \\
\text { industrial } \\
\text { sites }\end{array}$ & A & C-65\% & 7.0 & 4 \\
\hline PL & Niekłań 2 & $\underset{51^{\circ} 16^{\prime} 89.82^{\prime \prime}}{\mathrm{N}}$ & $\frac{E}{20^{\circ} 62^{\prime} 23.55^{\prime \prime}}$ & 283 & F3.111 & $\begin{array}{l}\text { Blackthorn- } \\
\text { bramble } \\
\text { scrub }\end{array}$ & $\mathrm{N}$ & $\begin{array}{l}\text { A-5\% } \\
\text { B-60\% } \\
\text { C- } 90 \%\end{array}$ & 7.0 & 3 \\
\hline
\end{tabular}


Table A1. Cont.

\begin{tabular}{|c|c|c|c|c|c|c|c|c|c|c|}
\hline \multirow{2}{*}{$\begin{array}{l}\text { Country } \\
\text { Code }\end{array}$} & \multirow{2}{*}{ Study Site } & \multicolumn{2}{|c|}{ GPS Coordinates } & \multirow{2}{*}{$\begin{array}{l}\text { Altitude } \\
\text { (m a.s.l.) }\end{array}$} & \multicolumn{3}{|c|}{ Habitat } & \multirow{2}{*}{$\begin{array}{c}\text { Plant } \\
\text { Cover* }\end{array}$} & \multirow{2}{*}{$\begin{array}{c}\text { Soil } \\
\mathrm{pH}\end{array}$} & \multirow{2}{*}{$\begin{array}{c}\text { Light } \\
\text { Availability ** }\end{array}$} \\
\hline & & Latitude & Longitude & & Code & Type & Origin & & & \\
\hline PL & Staporków, Miła & $\underset{51^{\circ} 13^{\prime} 74.93^{\prime \prime}}{\mathrm{N}}$ & $\frac{\mathrm{N}}{20^{\circ} 58^{\prime} 96.74^{\prime \prime}}$ & 267 & E5.43 & $\begin{array}{c}\text { Shady } \\
\text { woodland } \\
\text { edge fringes }\end{array}$ & $\mathrm{N}$ & $\begin{array}{l}\text { A-35\% } \\
\text { B-60\% } \\
\text { C-90\% }\end{array}$ & 7.0 & 2 \\
\hline PL & $\begin{array}{l}\text { Widawa } \\
\text { rivervalley, } \\
\text { between } \\
\text { Paniowice and } \\
\text { Szymanów }\end{array}$ & $\begin{array}{c}\mathrm{N} \\
51^{\circ} 12^{\prime} 11^{\prime \prime}\end{array}$ & $\begin{array}{c}E \\
16^{\circ} 59^{\prime} 09.37^{\prime \prime}\end{array}$ & 134 & E5.41 & $\begin{array}{l}\text { Screens or } \\
\text { veils of } \\
\text { perennial tall } \\
\text { herbs lining } \\
\text { watercourses }\end{array}$ & $\mathrm{SN}$ & C- $-90 \%$ & 7.5 & 4 \\
\hline PL & $\begin{array}{l}\text { Widawa } \\
\text { rivervalley, } \\
\text { between } \\
\text { Paniowice and } \\
\text { Szymanów }\end{array}$ & $\begin{array}{c}\mathrm{N} \\
50^{\circ} 56^{\prime} 22.4^{\prime \prime}\end{array}$ & $\begin{array}{c}\mathrm{E} \\
16^{\circ} 47^{\prime} 22.2^{\prime \prime}\end{array}$ & 148 & G1.11 & $\begin{array}{l}\text { Riverine Salix } \\
\text { woodland }\end{array}$ & $\mathrm{N}$ & $\begin{array}{l}\text { A- } 10 \% \\
\text { C- } 80 \%\end{array}$ & 8.0 & 4 \\
\hline PL & $\begin{array}{c}\text { Wrocław, } \\
\text { Zgorzelisko, bank } \\
\text { of Widawa river }\end{array}$ & $\begin{array}{c}\mathrm{N} \\
51^{\circ} 07^{\prime} 49.2^{\prime \prime}\end{array}$ & $\begin{array}{c}\mathrm{E} \\
17^{\circ} 07^{\prime} 44.0^{\prime \prime}\end{array}$ & 118 & E5.41 & $\begin{array}{c}\text { Screens or } \\
\text { veils of } \\
\text { perennial tall } \\
\text { herbs lining } \\
\text { watercourses }\end{array}$ & $\mathrm{N}$ & $\begin{array}{l}\text { A- } 10 \% \\
\text { B- } 50 \% \\
\text { C-70\% }\end{array}$ & 6.5 & 3 \\
\hline PL & $\begin{array}{c}\text { Kamieniec } \\
\text { Wrocławski, bank } \\
\text { of the Oder }\end{array}$ & $\begin{array}{c}\mathrm{N} \\
51^{\circ} 04^{\prime} 21.6^{\prime \prime}\end{array}$ & $\begin{array}{c}\mathrm{E} \\
17^{\circ} 10^{\prime} 11.9^{\prime \prime}\end{array}$ & 119 & E5.41 & $\begin{array}{l}\text { Screens or } \\
\text { veils of } \\
\text { perennial tall } \\
\text { herbs lining } \\
\text { watercourses }\end{array}$ & $\mathrm{N}$ & C- $90 \%$ & 7.0 & 4 \\
\hline PL & $\begin{array}{l}\text { Wrocław, Pilczyce, } \\
\text { bank of Ślęza river }\end{array}$ & $\underset{51^{\circ} 08^{\prime} 54.6^{\prime \prime}}{\mathrm{N}}$ & $\begin{array}{c}\mathrm{E} \\
16^{\circ} 57^{\prime} 22.6^{\prime \prime}\end{array}$ & 112 & E5.41 & $\begin{array}{l}\text { Screens or } \\
\text { veils of } \\
\text { perennial tall } \\
\text { herbs lining } \\
\text { watercourses }\end{array}$ & $\mathrm{N}$ & $\begin{array}{l}\text { B- } 40 \% \\
\text { C- } 50 \%\end{array}$ & 6.5 & 4 \\
\hline PL & $\begin{array}{l}\text { Joachimówko, } \\
\text { causeway of } \\
\text { StawTrójkątny }\end{array}$ & $\begin{array}{c}\mathrm{N} \\
51^{\circ} 32^{\prime} 20.9^{\prime \prime}\end{array}$ & $\begin{array}{c}\mathrm{E} \\
17^{\circ} 29^{\prime} 33.8^{\prime \prime}\end{array}$ & 113 & C3.2111 & $\begin{array}{c}\text { Freshwater } \\
\text { (Phragmites) } \\
\text { beds }\end{array}$ & $\mathrm{N}$ & C-100\% & 5.0 & 4 \\
\hline PL & Nowe Grodzisko & $\underset{51^{\circ} 33^{\prime} 25.3^{\prime \prime}}{\mathrm{N}}$ & $\begin{array}{c}E \\
17^{\circ} 21^{\prime} 26.1^{\prime \prime}\end{array}$ & 107 & $\mathrm{~J} 6.4$ & $\begin{array}{c}\text { Solid } \\
\text { agricultural } \\
\text { and } \\
\text { horticultural } \\
\text { waste }\end{array}$ & A & C-100\% & 7.5 & 4 \\
\hline PL & $\begin{array}{c}\text { Nowe Grodzisko, } \\
\text { bank of Barycz } \\
\text { river }\end{array}$ & $\begin{array}{c}\mathrm{N} \\
51^{\circ} 33^{\prime} 10.9^{\prime \prime}\end{array}$ & $\begin{array}{c}E \\
17^{\circ} 21^{\prime} 39.7^{\prime \prime}\end{array}$ & 106 & E5.11 & $\begin{array}{l}\text { Lowland } \\
\text { habitats } \\
\text { colonised by } \\
\text { tall } \\
\text { nitrophilous } \\
\text { herbs }\end{array}$ & A & $\begin{array}{l}\text { A-70\% } \\
\text { B-50\% } \\
\text { C-90\% }\end{array}$ & 6.0 & 2 \\
\hline PL & Nowe Brzesko & $\begin{array}{c}\mathrm{N} \\
50^{\circ} 07^{\prime} 16.8^{\prime \prime}\end{array}$ & $\begin{array}{c}\mathrm{E} \\
20^{\circ} 22^{\prime} 31.2^{\prime \prime}\end{array}$ & 186 & G1.111 & $\begin{array}{c}\text { Middle } \\
\text { European } \\
\text { white willow } \\
\text { forests }\end{array}$ & $\mathrm{N}$ & $\begin{array}{l}\text { A-40\% } \\
\text { B- } 40 \% \\
\text { C-40\% }\end{array}$ & 5.5 & 3 \\
\hline PL & CzapleMałe & $\underset{50^{\circ} 17^{\prime} 55.9^{\prime \prime}}{\mathrm{N}}$ & $\begin{array}{c}\mathrm{E} \\
19^{\circ} 57^{\prime} 18.8^{\prime \prime}\end{array}$ & 304 & E5.11 & $\begin{array}{l}\text { Lowland } \\
\text { habitats } \\
\text { colonised by } \\
\text { tall } \\
\text { nitrophilous } \\
\text { herbs } \\
\end{array}$ & A & C- $-100 \%$ & 5.2 & 4 \\
\hline PL & Pławowice & $\begin{array}{c}\mathrm{N} \\
50^{\circ} 10^{\prime} 25.6^{\prime \prime}\end{array}$ & $\begin{array}{c}\mathrm{E} \\
20^{\circ} 24^{\prime} 50.5^{\prime \prime} \\
\end{array}$ & 192 & F9.121 & $\begin{array}{c}\text { Almond } \\
\text { willow-osier } \\
\text { scrub }\end{array}$ & $\mathrm{N}$ & $\begin{array}{l}\text { A-40\% } \\
\text { B-50\% } \\
\text { C- } 40 \% \\
\end{array}$ & 5.5 & 4 \\
\hline PL & Odwiśle & $\begin{array}{c}\mathrm{N} \\
50^{\circ} 06^{\prime} 11.3^{\prime \prime}\end{array}$ & $\begin{array}{c}\mathrm{E} \\
20^{\circ} 19^{\prime} 56.4^{\prime \prime}\end{array}$ & 181 & $\mathrm{~J} 4.2$ & $\begin{array}{c}\text { Road } \\
\text { networks }\end{array}$ & A & C-100\% & 5.8 & 4 \\
\hline
\end{tabular}


Table A1. Cont.

\begin{tabular}{|c|c|c|c|c|c|c|c|c|c|c|}
\hline \multirow{2}{*}{$\begin{array}{l}\text { Country } \\
\text { Code }\end{array}$} & \multirow{2}{*}{ Study Site } & \multicolumn{2}{|c|}{ GPS Coordinates } & \multirow{2}{*}{$\begin{array}{l}\text { Altitude } \\
\text { (m a.s.l.) }\end{array}$} & \multicolumn{3}{|c|}{ Habitat } & \multirow{2}{*}{$\begin{array}{c}\text { Plant } \\
\text { Cover* }\end{array}$} & \multirow{2}{*}{$\begin{array}{c}\text { Soil } \\
\mathrm{pH}\end{array}$} & \multirow{2}{*}{$\begin{array}{c}\text { Light } \\
\text { Availability ** }\end{array}$} \\
\hline & & Latitude & Longitude & & Code & Type & Origin & & & \\
\hline PL & Kraków, Dąbie & $\underset{50^{\circ} 3^{\prime} 27^{\prime \prime}}{\mathrm{N}}$ & $\begin{array}{c}E \\
19^{\circ} 50^{\prime} 35^{\prime \prime}\end{array}$ & 195 & G1.1 & $\begin{array}{l}\text { Riparian and } \\
\text { gallery } \\
\text { woodland }\end{array}$ & $\mathrm{N}$ & $\begin{array}{l}\text { A-75\% } \\
\text { B- } 15 \% \\
\text { C-30\% } \\
\end{array}$ & 7.0 & 3 \\
\hline PL & Modlniczka & $\underset{50^{\circ} 11^{\prime} 46^{\prime \prime}}{\mathrm{N}}$ & $\begin{array}{c}\mathrm{E} \\
19^{\circ} 48^{\prime} 50^{\prime \prime}\end{array}$ & 179 & I2.2 & $\begin{array}{c}\text { Small-scale } \\
\text { ornamental } \\
\text { and domestic } \\
\text { garden areas }\end{array}$ & A & $\begin{array}{l}\text { A- } 10 \% \\
\text { B- } 40 \% \\
\text { C-70\% }\end{array}$ & 7.0 & 3 \\
\hline PL & Czajowice & $\begin{array}{c}\mathrm{N} \\
50^{\circ} 11^{\prime} 18^{\prime \prime}\end{array}$ & $\begin{array}{c}\mathrm{E} \\
19^{\circ} 48^{\prime} 18^{\prime \prime}\end{array}$ & 183 & $\mathrm{~J} 4.2$ & $\begin{array}{c}\text { Road } \\
\text { networks }\end{array}$ & A & C- $95 \%$ & 7.0 & 4 \\
\hline PL & $\begin{array}{c}\text { Kraków, Nowa } \\
\text { Huta } 1\end{array}$ & $\begin{array}{c}\mathrm{N} \\
50^{\circ} 04^{\prime} 08.81^{\prime \prime}\end{array}$ & $\begin{array}{c}\mathrm{E} \\
20^{\circ} 01^{\prime} 53.51^{\prime \prime}\end{array}$ & 198 & E2.7 & $\begin{array}{c}\text { Unmanaged } \\
\text { mesic } \\
\text { grassland }\end{array}$ & A & $\begin{array}{l}\mathrm{B} \leq 5 \% \\
\mathrm{C}-100 \%\end{array}$ & 6.5 & 4 \\
\hline PL & $\begin{array}{c}\text { Kraków, Nowa } \\
\text { Huta } 2\end{array}$ & $\begin{array}{c}\mathrm{N} \\
50^{\circ} 04^{\prime} 04.0^{\prime \prime}\end{array}$ & $\begin{array}{c}\mathrm{E} \\
20^{\circ} 02^{\prime} 24.4^{\prime \prime}\end{array}$ & 199 & E3.4 & $\begin{array}{l}\text { Moist or wet } \\
\text { eutrophic and } \\
\text { mesotrophic } \\
\text { grassland }\end{array}$ & $\mathrm{SN}$ & $\begin{array}{l}\mathrm{B} \leq 5 \% \\
\mathrm{C}-100 \%\end{array}$ & 6.5 & 4 \\
\hline PL & Strzyżów & $\underset{49^{\circ} 51^{\prime} 52.3^{\prime \prime}}{\mathrm{N}}$ & $\begin{array}{c}\mathrm{E} \\
21^{\circ} 46^{\prime} 45.4^{\prime \prime}\end{array}$ & 220 & G1.11 & $\begin{array}{l}\text { Riverine Salix } \\
\text { woodland }\end{array}$ & $\mathrm{N}$ & $\begin{array}{r}\text { A- } 60 \% \\
\text { B- } 40 \% \\
\text { C- }-100 \% \\
\end{array}$ & 7.0 & 2 \\
\hline PL & Dobrzechów & $\underset{49^{\circ} 52^{\prime} 04.4^{\prime \prime}}{\mathrm{N}}$ & $\underset{21^{\circ} 44^{\prime} 51.9^{\prime \prime}}{E}$ & 219 & G1.2 & $\begin{array}{l}\text { Mixed } \\
\text { riparian } \\
\text { floodplain } \\
\text { and gallery } \\
\text { woodland }\end{array}$ & $\mathrm{N}$ & $\begin{array}{r}\text { B- } 60 \% \\
\text { C- } 100 \%\end{array}$ & 7.0 & 3 \\
\hline PL & Wiśniowa & $\underset{49^{\circ} 51^{\prime} 40.4^{\prime \prime}}{\mathrm{N}}$ & $\begin{array}{c}\mathrm{E} \\
21^{\circ} 39^{\prime} 33.7^{\prime \prime}\end{array}$ & 227 & C 3.26 & $\begin{array}{c}\text { Phalaris } \\
\text { arundinacea } \\
\text { beds }\end{array}$ & $\mathrm{N}$ & C- $-100 \%$ & 7.0 & 4 \\
\hline PL & $\begin{array}{l}\text { Wielopole } \\
\text { Skrzyńskie }\end{array}$ & $\begin{array}{c}\mathrm{N} \\
49^{\circ} 56^{\prime} 50.2^{\prime \prime}\end{array}$ & $\begin{array}{c}\mathrm{E} \\
21^{\circ} 37^{\prime} 00.6^{\prime \prime}\end{array}$ & 254 & $\mathrm{~J} 4.2$ & $\begin{array}{c}\text { Road } \\
\text { networks }\end{array}$ & $\mathrm{A}$ & C- $-80 \%$ & 6.5 & 4 \\
\hline PL & Broniszów & $\begin{array}{c}\mathrm{N} \\
49^{\circ} 59^{\prime} 18.6^{\prime \prime}\end{array}$ & $\begin{array}{c}\mathrm{E} \\
21^{\circ} 33^{\prime} 41.3^{\prime \prime}\end{array}$ & 218 & $\mathrm{~J} 4.2$ & $\begin{array}{c}\text { Road } \\
\text { networks }\end{array}$ & A & C- $-100 \%$ & 7.0 & 4 \\
\hline PL & Okonin & $\underset{50^{\circ} 01^{\prime} 49.52^{\prime \prime}}{\mathrm{N}}$ & $\underset{21^{\circ} 32^{\prime} 56.03^{\prime \prime}}{\mathrm{E}}$ & 199 & G1.11 & $\begin{array}{l}\text { Riverine Salix } \\
\text { woodland }\end{array}$ & $\mathrm{N}$ & $\begin{array}{c}\text { A- } 60 \% \\
\text { B-50\% } \\
\text { C- } 100 \%\end{array}$ & 7.0 & 3 \\
\hline PL & $\begin{array}{l}\text { Rzeszów, the } \\
\text { Rzeszów reservoir }\end{array}$ & $\underset{49^{\circ} 59^{\prime} 54.2^{\prime \prime}}{\mathrm{N}}$ & $\begin{array}{c}\mathrm{E} \\
21^{\circ} 58^{\prime} 44.6^{\prime \prime}\end{array}$ & 199 & G1.11 & $\begin{array}{c}\text { Riverine Salix } \\
\text { woodland }\end{array}$ & $\mathrm{N}$ & $\begin{array}{c}\text { A- } 60 \% \\
\text { B- } 50 \% \\
\text { C- }-100 \%\end{array}$ & 7.0 & 2 \\
\hline PL & $\begin{array}{c}\text { Rzeszów, } \\
\text { boulevardsnear- } \\
\text { Wisłokriver }\end{array}$ & $\underset{50^{\circ} 01^{\prime} 24.9^{\prime \prime}}{\mathrm{N}}$ & $\begin{array}{c}\mathrm{E} \\
21^{\circ} 00^{\prime} 01.6^{\prime \prime}\end{array}$ & 193 & $\mathrm{~J} 4.2$ & $\begin{array}{c}\text { Road } \\
\text { networks }\end{array}$ & A & $\begin{array}{c}\text { B-5\% } \\
\text { C- }-100 \%\end{array}$ & 7.0 & 4 \\
\hline SK & $\begin{array}{l}\text { Budca, near Hron } \\
\text { River } 1\end{array}$ & $\underset{48^{\circ} 33^{\prime} 44.7^{\prime \prime}}{\mathrm{N}}$ & $\begin{array}{c}\mathrm{E} \\
19^{\circ} 02^{\prime} 36.2^{\prime \prime}\end{array}$ & 235 & G1.1 & $\begin{array}{c}\text { Riparian and } \\
\text { gallery } \\
\text { woodland }\end{array}$ & $\mathrm{N}$ & $\begin{array}{l}\text { B- } 90 \% \\
\text { C- } 60 \%\end{array}$ & 7.0 & 3 \\
\hline SK & $\begin{array}{c}\text { Budca, near Hron } \\
\text { River } 2\end{array}$ & $\underset{48^{\circ} 33^{\prime} 46.7^{\prime \prime}}{\mathrm{N}}$ & $\begin{array}{c}\mathrm{E} \\
19^{\circ} 02^{\prime} 26.3^{\prime \prime}\end{array}$ & 233 & G1.1 & $\begin{array}{l}\text { Riparian and } \\
\text { gallery } \\
\text { woodland }\end{array}$ & $\mathrm{N}$ & $\begin{array}{l}\text { B- } 5 \% \\
\text { C- } 90 \%\end{array}$ & 7.0 & 4 \\
\hline SK & $\begin{array}{c}\text { Budca, near Hron } \\
\text { River } 3\end{array}$ & $\underset{48^{\circ} 33^{\prime} 47.7^{\prime \prime}}{\mathrm{N}}$ & $\begin{array}{c}\mathrm{E} \\
19^{\circ} 02^{\prime} 22.8^{\prime \prime}\end{array}$ & 250 & G1.1 & $\begin{array}{c}\text { Riparian and } \\
\text { gallery } \\
\text { woodland }\end{array}$ & $\mathrm{N}$ & $\begin{array}{c}\text { A- } 10 \% \\
\text { B-5\% } \\
\text { C } \geq 95 \%\end{array}$ & 6.0 & 4 \\
\hline SK & $\begin{array}{l}\text { Budca, near Hron } \\
\text { River } 4\end{array}$ & $\underset{48^{\circ} 33^{\prime} 42.1^{\prime \prime}}{\mathrm{N}}$ & $\begin{array}{c}\mathrm{E} \\
1^{\circ} 01^{\prime} 24.8^{\prime \prime}\end{array}$ & 255 & G1.1 & $\begin{array}{c}\text { Riparian and } \\
\text { gallery } \\
\text { woodland }\end{array}$ & $\mathrm{N}$ & $\begin{array}{c}\text { A-60\% } \\
\text { B-5\% } \\
C \geq 95 \%\end{array}$ & 6.5 & 3 \\
\hline SK & Slatina River1 & $\underset{48^{\circ} 33^{\prime} 47.0^{\prime \prime}}{\mathrm{N}}$ & $\begin{array}{c}E \\
19^{\circ} 06^{\prime} 38.2^{\prime \prime}\end{array}$ & 271 & G1.1 & $\begin{array}{l}\text { Riparian and } \\
\text { gallery } \\
\text { woodland }\end{array}$ & $\mathrm{N}$ & $\begin{array}{l}\mathrm{A} \geq 5 \% \\
\mathrm{~B}-80 \% \\
\mathrm{C} 95 \%\end{array}$ & 7.0 & 2 \\
\hline
\end{tabular}


Table A1. Cont.

\begin{tabular}{|c|c|c|c|c|c|c|c|c|c|c|}
\hline \multirow{2}{*}{$\begin{array}{l}\text { Country } \\
\text { Code }\end{array}$} & \multirow{2}{*}{ Study Site } & \multicolumn{2}{|c|}{ GPS Coordinates } & \multirow{2}{*}{$\begin{array}{l}\text { Altitude } \\
\text { (m a.s.1.) }\end{array}$} & \multicolumn{3}{|c|}{ Habitat } & \multirow{2}{*}{$\begin{array}{l}\text { Plant } \\
\text { Cover* }\end{array}$} & \multirow{2}{*}{$\begin{array}{l}\text { Soil } \\
\mathrm{pH}\end{array}$} & \multirow{2}{*}{$\begin{array}{c}\text { Light } \\
\text { Availability }\end{array}$} \\
\hline & & Latitude & Longitude & & Code & Type & Origin & & & \\
\hline SK & Slatina River2 & $\underset{48^{\circ} 33^{\prime} 46.3^{\prime \prime}}{\mathrm{N}}$ & $\begin{array}{c}\mathrm{E} \\
19^{\circ} 06^{\prime} 36.6^{\prime \prime}\end{array}$ & 269 & G1.1 & $\begin{array}{l}\text { Riparian and } \\
\text { gallery } \\
\text { woodland }\end{array}$ & $\mathrm{N}$ & $\begin{array}{c}A-70 \% \\
B \geq 5 \% \\
C \geq 95 \%\end{array}$ & 7.0 & 3 \\
\hline
\end{tabular}

* Vegetation layers: A-tree layer, B-shrub layer, C-herb layer. ** The descriptive scale of light availability at ground level: 1 -full shade (0-20\% of light availability); 2 -substantial shade (21-40\% of light availability); 3-moderate shade (41-60\% of light availability); 4-low shade (61-80\% of light availability); 5-full sun (81-100\% of light availability). The scale was created by calculating the mean plant coverage in A, B, and C layers, and then subtracting the obtained value from $100 \%$ insolation.

\section{References}

1. Gianoli, E. The behavioural ecology of climbing plants. AoB Plants 2015, 7, plv013. [CrossRef] [PubMed]

2. Gentry, A.H. The distribution and evolution of climbing plants. In The Biology of Vines; Putz, F.E., Mooney, H.A., Eds.; Cambridge University Press: Cambridge, UK, 1991; pp. 3-49.

3. Putz, F.E. Vine Ecology. 2012. Available online: http://www.ecology.info/vines.htm (accessed on 7 May 2021).

4. Darwin, C.R. On the movements and habits of climbing plants. J. Linn. Soc. Bot. 1865, 9, 1-118. [CrossRef]

5. Addo-Fordjour, P.; Anning, A.K.; Atakora, E.A.; Agyei, P.S. Diversity and distribution of climbing plants in a semi-deciduous rain forest, KNUST Botanic Garden, Ghana. Int. J. Bot. 2008, 4, 186-195. [CrossRef]

6. Bitomský, M.; Mládková, P.; Cimalová, Š.; Mládek, J. Herbaceous climbers in herbaceous systems are shade-tolerant and magnesium-demanding. J. Veg. Sci. 2019, 30, 799-808. [CrossRef]

7. Stansbury, C.D.; Batchelor, K.L.; Morin, L.; Woodburn, T.L.; Scott, J.K. Standardized support to measure biomass and fruit production by the invasive climber (Asparagus asparagoides). Weed Technol. 2007, 21, 820-824. [CrossRef]

8. $\mathrm{Hu}, \mathrm{L} . ; \mathrm{Li}, \mathrm{M}$. Climbing capacity of the invasive vine Mikania micrantha Kunth on vertical artificial poles. Biol. Invasions 2014, 16, 295-302. [CrossRef]

9. Grašič, M.; Piberčnik, M.; Zelnik, I.; Abram, D.; Gaberščik, A. Invasive alien vines affect leaf traits of riparian woody vegetation. Water 2019, 11, 2395. [CrossRef]

10. Burnham, R.J.; Santanna, C.V. Distribution, diversity, and traits of native, exotic, and invasive climbing plants in Michigan. Brittonia 2015, 67, 350-370. [CrossRef]

11. Yang, S.-I.; Walters, T.W. Ethnobotany and the economic role of the Cucurbitaceae of China. Econ. Bot. 1992, 46, 349-367. [CrossRef]

12. Rahman, A.H.M.M.; Anisuzzaman, M.; Ferdous, A.; Rafiul Islam, A.K.M.; Naderuzzaman, A.T.M. Study of nutritive value and medicinal uses of cultivated cucurbits. J. Appl. Sci. Res. 2008, 4, 555-558.

13. Kujawska, M.; Svanberg, I. From medicinal plant to noxious weed: Bryonia alba L. (Cucurbitaceae) in northern and eastern Europe. J. Ethnobiol. Ethnomed. 2019, 15, 22. [CrossRef]

14. Zhao, F.X.; Yan, S.H.; Li, M.H.; Liu, X.Y.; Zhang, X.W.; Cao, Y.; Zhao, H. Adaptive strategies of structures that enhance invasion in Sicyos angulatus. Not. Bot. HortiAgrobo. 2019, 47, 1323-1330. [CrossRef]

15. Larché, J.F. Sicyos angulatus, a new weed for corn crops in SW France. Phytoma 2004, 571, 19-22.

16. Kil, J.H.; Kong, H.Y.; Koh, K.S.; Kim, J.M. Management of Sicyos angulata spread in Korea. In: Neobiota. From Ecology to Conservation. In Proceedings of the 4th European Conference on Biological Invasions, Vienna, Austria, 27-29 September 2006. BfN-Skripten 184:170.

17. EPPO. Sicyos angulatus. 2010. Available online: https://onlinelibrary.wiley.com/doi/10.1111/j.1365-2338.2010.02415.x (accessed on 15 November 2010). [CrossRef]

18. Starr, F.; Starr, K.; Loope, L. Coccinia grandis-Hawaiian Ecosystems at Risk Project (HEAR). Available online: http://www.hear. org/starr/hiplants/reports/pdf/coccinia_grandis.pdf (accessed on 8 October 2020).

19. Muniappan, R.; Reddy, G.V.P.; Raman, A. Coccinia grandis (L.) Voigt (Cucurbitaceae). In Biological Control of Tropical Weeds Using Arthropods; Muniappan, R., Ed.; Cambridge University Press: Cambridge, UK, 2009; pp. 175-182.

20. Chun, M.E. Biology and host specificity of Melittia oedipus (Lepidoptera: Sesiidae), a biological control agent of Coccinia grandis (Cucurbitaceae). Proc. Hawaii Entomol. Soc. 2001, 35, 85-93.

21. Kuluev, B.R.; Shvets, D.Y.; Golovanov, Y.M.; Probatova, N.S. Thladiantha dubia (Cucurbitaceae) in the Republic of Bashkortostan as a dangerous weed with high invasive potential. Russ. J. Biol. Invasions 2019, 10, 160-170. [CrossRef]

22. Early, R.; Bradley, B.A.; Dukes, J.S.; Lawler, J.J.; Olden, J.D.; Blumenthal, D.M.; Gonzalez, P.; Grosholz, E.D.; Ibañez, I.; Miller, L.P.; et al. Global threats from invasive alien species in the twenty-first century and national response capacities. Nat. Commun. 2016, 7, 12485. [CrossRef]

23. Celesti-Grapow, L.; Ricottam, C. Plant invasion as an emerging challenge for the conservation of heritage sites: The spread of ornamental trees on ancient monuments in Rome, Italy. Biol. Invasions 2021, 23, 1191-1206. [CrossRef] 
24. Oh, M.; Heo, Y.; Lee, E.J.; Lee, H. Major environmental factors and traits of invasive alien plants determine their spatial distribution: A case study in Korea. J. Ecol. Environ. 2021, 45, 18. [CrossRef]

25. Lembrechts, J.J.; Rossi, E.; Milbau, A.; Nijs, I. Habitat properties and plant traits interact as drivers of non- native plant species' seed production at the local scale. Ecol. Evol. 2018, 8, 4209-4223. [CrossRef]

26. Johnson, J.S.; Cantrell, R.S.; Cosner, C.; Hartig, F.; Hastings, A.; Rogers, H.S.; Schupp, E.W.; Shea, K.; Teller, B.J.; Yu, X.; et al. Rapid changes in seed dispersal traits may modify plant responses to global change. AoB PLANTS 2019, 11, plz020. [CrossRef]

27. DiTommaso, A.; Stokes, C.A.; Cordeau, S.; Milbrath, L.R.; Whitlow, T.H. Seed-dispersal ability of the invasive perennial vines Vincetoxicum nigrum and Vincetoxicum rossicum. Invasive Plant Sci. Manag. 2018, 11, 10-19. [CrossRef]

28. Tokarska-Guzik, B. The Establishment and Spread of Alien Plant Species (Kenophytes) in the Flora of Poland; Wydawnictwo Uniwersytetu Śląskiego: Katowice, Poland, 2005.

29. Klotz, S. Echinocystis lobata (Michx.) Torr. \& Gray., wild cucumber (Cucurbitaceae, Magnoliophyta). In Handbook of Alien Species in Europe; Hulme, P.E., Nentwig, W., Pyšek, P., Vilà, M., Eds.; Invading Nature-Springer Series in Invasion Ecology; Springer: Dordrecht, The Netherlands, 2009; p. 347.

30. Priede, A. Distribution of some invasive alien plant species in riparian habitats in Latvia. Bot. Lith. 2008, 14, 137-150.

31. Rutkovska, S.; Pučka, I.; Novicka, I.; Evarts-Bunders, P. Relationship of geographic distribution of the most characteristical invasive plant species in habitats adjacent to the river Daugava within the territory of Daugavpils city. Acta Biol. Univ. Daugavp. 2011, 11, 163-175.

32. Dylewski, Ł.; Myczko, Ł.; Pearson, D.E. Native generalist consumers interact strongly with seeds of the invasive wild cucumber (Echinocystis lobata). NeoBiota 2019, 53, 25-39. [CrossRef]

33. European Environmental Agency. The EUNIS Habitat Classification. Available online: https://www.eea.europa.eu/data-andmaps / data/eunis-habitat-classification (accessed on 25 February 2020).

34. Choate, H.A. Dormancy and germination in seeds of Echinocystis lobata. Am. J. Bot. 1940, 27, 156-160. [CrossRef]

35. Silvertown, J. Survival, fecundity and growth of wild cucumber, Echinocystis lobata. J. Ecol. 1985, 73, 841-849. [CrossRef]

36. Stocking, K.M. Some considerations of the genera Echinocystis and Echinopepon in the United States and northern Mexico. Madroño 1955, 13, 84-100.

37. Nature Manitoba; Reaume, T. Wild Cucumber Echinocystis lobata. 2010. Available online: https://www.naturemanitoba.ca/sites/ default/files/WildCucumber.pdf (accessed on 7 May 2021).

38. Bagi, I.; Böszörményi, A. Wild cucumber (Echinocystis lobata Torr. et Gray). In The Most Important Invasive Plants in Hungary; Botta-Dukát, Z., Balogh, L., Eds.; Institute of Ecology and Botany, Hungarian Academy of Sciences: Vácrátót, Hungary, 2008; pp. 103-114.

39. Randall, R.P. A Global Compendium of Weeds, 3rd ed.; Randall RP: Perth, WE, Australia, 2017.

40. Vasić, O. Echinocystis lobata (Michx) Torrey et A. Gray in Serbia. Acta Bot. Croat. 2005, 64, 369-373.

41. Botta-Dukát, Z. Invasion of alien species to Hungarian (semi-) natural habitats. Acta Bot. Hung. 2008, 50, 219-227. [CrossRef]

42. Towpasz, K.; Stachurska-Swakon, A. Alder-ash and willow communities and their diversity in the PogórzeStrzyżowskie foothills (Western Carpathians). Acta Soc. Bot. Pol. 2008, 77, 327-338.

43. Towpasz, K.; Stachurska-Swakon, A. The analysis of the forest flora of the Strzyżowskie Foothills from the perspective of presence of anthropogenic species. Acta Univ. Lodz. Folia Biol. Oecol. 2011, 7, 99-110. [CrossRef]

44. Towpasz, K.; Stachurska-Swakoń, A. Occurrence of alien species in the agriculture landscape: A case of Proszowice Plateau (Southern Poland). Ann. Univ. Paedagog. Crac. Stud. Nat. 2018, 3, 7-21. [CrossRef]

45. Zelnik, I. The presence of invasive alien plant species in different habitats: Case study from Slovenia. Acta Biol. Slov. 2012, 55, 25-38.

46. Kołaczkowska, E. Kolczurka klapowana Echinocystis lobata (F. Michx.) Torrey\& A. Gray. In Inwazyjne gatunki roślin w Kampinoskim Parku Narodowym i jego sasiedztwie. Kampinoski Park Narodowy; Otręba, A., Michalska-Hejduk, D., Eds.; Kampinoski Park Narodowy: Izabelin, Poland, 2014; pp. 37-40.

47. Gerrath, J.M.; Guthrie, T.B.; Zitnak, T.A.; Posluszny, U. Development of the axillary bud complex in Echinocystislobata (Cucurbitaceae): Interpreting the Cucurbitaceoustendril. Am. J. Bot. 2008, 95, 773-781. [CrossRef] [PubMed]

48. Kovach, W.L. MVSP-A MultiVariate Statistical Package for Windows ver. 3; Kovach Computing Services: Pentraeth, UK, 2010.

49. Van Emden, H. Statistics for Terrified Biologists; Blackwell Publishing: Hoboken, NJ, USA, 2008.

50. Rolbiecki, R.; Rolbiecki, S.; Figas, A.; Wichrowska, D.; Jagosz, B.; Ptach, W. The efficiency of drip fertigation in cultivation of winter squash 'Gomez' on the very light soil. Infrastruct. Ecol. Rural. Areas 2017, 3, 1201-1211.

51. Spjut, R.W. A Systematic Treatment of the Fruit Types. 2015. Available online: http://www.worldbotanical.com/fruit_types.htm (accessed on 7 May 2021).

52. Nerson, H. Seed production and germinability of cucurbit crops. Seed Sci. Biotech. 2007, 1, 1-10.

53. Oliveira, E.C.; de Carvalho, J.A.; da Silva, W.G.; Rezende, F.C.; de Almeida, W.F. Effects of water deficit in two phenological stages on production of japanese cucumber cultived in greenhouse. Eng. Agríc. 2011, 31, 676-686. [CrossRef]

54. Maynard, L. Cucurbit crop growth and development. In Indiana CCA Conference Proceedings; Purdue University: West Lafayette, IN, USA, 2007.

55. Esbenshade, W.R.; Curran, W.S.; Roth, G.W.; Hartwig, N.L.; Orzolek, M.D. Effect of establishment date and crop competition on burcucumber fecundity. Weed Sci. 2001, 49, 524-527. [CrossRef] 
56. Zelnik, I.; Klenovsek, V.M.; Gaberscik, A. Complex undisturbed riparian zones are resistant to colonisation by invasive alien plant species. Water 2020, 12, 345. [CrossRef]

57. Obidziński, A.; Mędrzycki, P.; Kołaczkowska, E.; Ciurzycki, W.; Marciszewska, K. Do David and Goliathplay the same game? Explanation of the abundance of rare and frequent invasive alien plants in urban woodlands in Warsaw, Poland. PLoS ONE 2016, 11, e0168365. [CrossRef]

58. Kazinczi, G.; Horváth, J.; Hunyadi, K. Germination biology and virus susceptibility of wild cucumber (Echinocystis lobata Torr. et Gray). Növénytermelés 1998, 47, 645-654.

59. Lukatkin, A.S.; Tyutyaev, E.V.; Sharkaeva, E.S.; Lukatkin, A.A.; Teixeira da Silva, J.A. Mild abiotic stresses have different effects on chlorophyll fluorescence parameters in leaves of young woody and herbaceous invasive plants. Acta Physiol. Plant 2017, 39, 20. [CrossRef]

60. Önen, H.; Farooq, S.; Tad, S.; Özaslan, C.; Gunal, H.; Chauhan, B.S. The influence of environmental factors on germination of burcucumber (Sicyos angulatus) seeds: Implications for range expansion and management. Weed Sci. 2018, 66, 494-501. [CrossRef]

61. Wyka, T.P.; Zadworny, M.; Mucha, J.; Żytkowiak, R.; Nowak, K.; Oleksyn, J. Species-specific responses of growth and biomass distribution to trellis availability in three temperate lianas. Trees 2019, 33, 921-932. [CrossRef]

62. Paul, G.S.; Yavitt, J.B. Tropical vine growth and the effects on forest succession: A review of the ecology and management of tropical climbing plants. Bot. Rev. 2011, 77, 11-30. [CrossRef]

63. Driedonks, N.; Rieu, I.; Vriezen, W.H. Breeding for plant heat tolerance at vegetative and reproductive stages. Plant Reprod. 2016, 29, 67-79. [CrossRef] [PubMed]

64. Kaushal, N.; Bhandarik, K.; Siddique, K.H.; Nayyar, H. Food crops face rising temperatures: An overview of responses, adaptive mechanisms, and approaches to improve heat tolerance. Cogent Food Agric. 2016, 2, 1134380. [CrossRef]

65. Janni, M.; Gullì, M.; Maestri, E.; Marmiroli, M.; Valliyodan, B.; Nguyen, H.T.; Marmiroli, N. Molecular and genetic bases of heat stress responses in crop plants and breeding for increased resilience and productivity. J. Exp. Bot. 2020, 71, 3780-3802. [CrossRef]

66. Jing, H.-C.; Bergervoet, J.H.W.; Jalink, H.; Klooster, M.; Du, S.-L.; Bino, R.J.; Hilhorst, H.W.M.; Groot, S.P.C. Cucumber (Cucumis sativus L.) seed performance as influenced by ovary and ovule position. Seed Sci. Res. 2000, 10, 435-445. [CrossRef]

67. Nerson, H. Fruit-set order affects seed yield and germinability in melon (Cucumis melo L.). J. Hortic. Sci. Biotechnol. 2004, 79, 985-990. [CrossRef]

68. Nerson, H. Does fruit number per plant, or fruit-set order affect seed yield and quality in cucumber? J. Hortic. Sci. Biotechnol. 2008, 83, 160-164. [CrossRef]

69. Nerson, H.; Paris, H.S. Effects of fruit age, fermentation and storage on germination of cucurbit seeds. Sci. Hortic. 1988, 35, 15-26. [CrossRef]

70. Khan, A.S.M.M.R.; Kabir, M.Y.; Alam, M.M. Variability, correlation path analysis of yield and yield components of pointed gourd J. Agric. Rural. Dev. 2009, 7, 93-98. [CrossRef]

71. Tanaka, K.; Duong, T.-T.; Yamashita, H.; Heng, S.L.; Sophany, S.; Kato, K. Collection of cucurbit crops (Cucurbitaceae) from Eastern Cambodia. AREIPGR 2017, 32, 109-137.

72. Sari, N.; Solmaz, I.; Pamuk, S.; Cetin, B.; Gocmen, M.; Simsek, I. Fruit and seed size in some mini watermelon lines. Acta Hortic. 2017, 1151, 109-114. [CrossRef]

73. Nerson, H. Plant density, fruit length and fruit type affect seed yield and quality in cucumber. Adv. Hortic. Sci. 2005, 19, 206-212.

74. Barzegar, R.; Houshmand, S.; Peyvast, G.H. Relationship between seed yield and some of fruit traits in Iranian squash (Cucurbita pepo L.) accissions. J. Hortic. Sci. 2015, 29, 142-149. [CrossRef]

75. Dylewski, Ł.; Maćkowiak, Ł.; Myczko, Ł. Physical defence of the wild cucumber Echinocystis lobata in an invasive range changing seed removal by rodents. Plant Ecol. 2018, 219, 863-873. [CrossRef]

76. Golivets, M. Variation in quantitative seed traits of Echinocystis lobata (Michx.) Torr. et A. Gray (Cucurbitaceae). Mod. Phytomorphol. 2014, 6, 43-44.

77. Louda, S.M. Distribution ecology: Variation in plant recruitment over a gradient in relation to insect seed predation. Ecol. Monogr. 1982, 52, 25-41. [CrossRef]

78. Maron, J.L.; Gardner, S.N. Consumer pressure, seed versus safe-site limitation, and plant population dynamics. Oecologia 2000, 124, 260-269. [CrossRef] [PubMed]

79. Maron, J.L.; Crone, E. Herbivory: Effects on plant abundance, distribution and population growth. Proc. Royal Soc. B 2006, 273, 2575-2584. [CrossRef] [PubMed]

80. Stachurska-Swakoń, A.; Barabasz-Krasny, B.; Klasa, A.; Palaczyk, A. Reduced plant fitness by pre-dispersal seed predation in the threatened plant species Cirsium decussatum. Seed Sci. Res. 2018, 28, 123-130. [CrossRef]

81. Sinnott-Armstrong, M.A.; Downie, A.E.; Federman, S.; Valido, A.; Jordano, P.; Donoghue, M.J. Global geographic patterns in the colours and sizes of animal-dispersed fruits. Glob. Ecol. Biogeogr. 2018, 27, 1-13. [CrossRef]

82. Richardson, D.M.; Allsopp, N.; D’Antonio, C.M.; Milton, S.J.; Rejmánek, M. Plant invasions—The role of mutualisms. Biol. Rev. 2000, 75, 65-93. [CrossRef]

83. Zając, A.; Tokarska-Guzik, B.; Zając, M. The role of rivers and streams in the migration of alien plants into the Polish Carpathians. Biodiv. Res.Conserv. 2011, 23, 43-56. [CrossRef]

84. Moles, A.T.; Westoby, M. Latitude, seed predation and seed mass. J. Biogeogr. 2003, 30, 105-128. [CrossRef] 
85. Moles, A.T.; Ackerly, D.D.; Tweddle, J.C.; Dickie, J.B.; Smith, R.; Leishman, M.R.; Mayfield, M.M.; Pitman, A.; Wood, J.T.; Westoby, M. Global patterns in seed size. Glob. Ecol. Biogeogr. 2007, 16, 109-116. [CrossRef]

86. Pluess, A.R.; Schutz, W.; Stocklin, J. Seed weight increases with altitude in the Swiss Alps between related species but not among populations of individual species. Oecologia 2005, 144, 55-61. [CrossRef]

87. Guo, H.; Mazer, S.J.; Du, G. Geographic variation in seed mass within and among nine species of Pedicularis (Orobanchaceae): Effects of elevation, plant size and seed number per fruit. J. Ecol. 2010, 98, 1232-1242. [CrossRef]

88. Wang, Y.; Wang, J.; Lai, L.; Jiang, L.; Zhuang, P.; Zhang, L. Geographic variation in seed traits within and among forty-two species of Rhododendron (Ericaceae) on the Tibetan plateau: Relationships with altitude, habitat, and phylogeny. Ecol. Evol. 2014, 4, 1913-1923. [CrossRef]

89. Olejniczak, P.; Czarnoleski, M.; Delimat, A.; Majcher, B.M.; Szczepka, K. Seed size in mountain herbaceous plants changes with elevation in a species-specific manner. PLOS ONE 2018, 13, e0199224. [CrossRef] [PubMed]

90. Fenner, M.; Thompson, K. Ecology of Seeds; Cambridge University Press: Cambridge, UK, 2005.

91. Guo, Y.; Gao, M.; Liang, X.; Xu, M.; Liu, X.; Zhang, Y.; Liu, X.; Liu, J.; Gao, Y.; Qu, S.; et al. Quantitative trait loci for seed size variation in cucurbits-A review. Front. Plant. Sci. 2020, 11, 304. [CrossRef] [PubMed]

92. Paris, H.S.; Nerson, H. Seed dimensions in the subspecies and cultivar-groups of Cucurbita pepo. Gen. Resour. Crop Evol. 2003, 50, 615-625. [CrossRef]

93. Rindyastuti, R.; Hapsari, L.; Byun, C. Comparison of ecophysiological and leaf anatomical traits of native and invasive plant species. J. Ecol. Environ. 2021, 45, 4. [CrossRef]

94. Molina-Montenegro, M.A.; Acuña-Rodríguez, I.S.; Flores, T.S.M.; Hereme, R.; Lafon, A.; Atala, C.; Torres-Díaz, C. Is the Success of Plant Invasions the Result of Rapid Adaptive Evolution in Seed Traits? Evidence from a Latitudinal Rainfall Gradient. Front. Plant. Sci. 2018, 9, 208. [CrossRef] [PubMed] 